\title{
Source of arsenic based on geological and hydrogeochemical properties of geothermal systems in Western Turkey
}

\author{
Alper Baba ${ }^{\mathrm{a}, *}$, Hasan Sözbilir ${ }^{\text {b }}$ \\ a İmir Institute of Technology, Geothermal Energy Research and Application Center, Urla, Izmir, Turkey \\ ${ }^{\mathrm{b}}$ Dokuz Eylul University, Engineering Faculty, Department of Geological Engineering, Izmir, Turkey
}

\section{A R T I C L E I N F O}

Article history:

Accepted 12 June 2012

Available online 29 June 2012

Editor: J.D. Blum

\section{Keywords:}

Arsenic

Geothermal

Tectonics

Turkey

\begin{abstract}
A B S T R A C T
Turkey is an area of complex geology with active tectonics and high geothermal potential. Especially, the western part of Turkey is a region of abundant geothermal activity. Faults accommodating the deep circulation of hydrothermal fluids of meteoric origin are the primary means by which of geothermal systems are controlled in this region. Many of the thermal activities are related to the improved dilation on the $\sim \mathrm{E}-$ $\mathrm{W}$-strikes of the graben faults. This situation serves as a suitable environment for the presence of high levels of arsenic in geothermal water resources. The highest concentrations of naturally occurring aqueous arsenic (As) are found in certain types of geothermal waters, generally those related to major graben faults. In this regard, high arsenic concentrations in geothermal resources have been detected in Western Turkey, including but not limited to Biga Peninsulla, Gediz Graben, Kucuk, and Buyuk Menderes Graben with values ranging from 1 to $1419 \mathrm{ppb}$ in geothermal fluids. The thermal waters have surface temperatures of up to $100{ }^{\circ} \mathrm{C}$ and reservoir temperatures range from 150 to $248{ }^{\circ} \mathrm{C}$ in the Menderes Graben, from 120 to $287^{\circ} \mathrm{C}$ in the Gediz Graben, and from 153 to $174{ }^{\circ} \mathrm{C}$ in Biga Peninsula. Hydrogeochemically, the Menderes graben and Gediz Graben thermal waters are of the $\mathrm{Na}-\mathrm{HCO}_{3}, \mathrm{Ca}-\mathrm{HCO}_{3}$, and $\mathrm{Na}-\mathrm{SO}_{4}$ types, whereas some geothermal fluids such as those of Tuzla and Kestanbol in the Biga Peninsula, Çeşme, and Urla are of the Na-Cl type.
\end{abstract}

(c) 2012 Elsevier B.V. All rights reserved.

\section{Introduction}

Occurrence of arsenic (As) in groundwater and its impacts on millions of individuals worldwide is a serious environmental health issue. The sources of As are both natural and anthropogenic and affect different regions of the world both on a local, as well as on a regional scale (Van Halem et al., 2009; Bundschuh et al., 2010; Gunduz et al., 2010a). In many countries such as India and Bangladesh As is naturally found in the subsurface strata within volcanic and sedimentary formations as well as in areas of geothermal systems related to tectonic activity. For well over 100 years, high As concentrations have been reported for many thermal waters and fumaroles (Nordstrom, 2010). Arsenic concentrations at the geothermal field, El Tatio (North Chile), Copper River (USA), Yellowstone National Park (USA), and Los Humeros (Mexico), have been reported to be as high as $48 \mathrm{ppm}, 48.2 \mathrm{ppm}, 15 \mathrm{ppm}$, and 74 ppm respectively (Ellis and Mahon, 1967; Motyka et al., 1989; Ball et al., 1998; Gonzalez-Partida et al., 2001; Romero et al., 2003). Generally, a high concentration of As is found where there is sulfide-mineral precipitation as a result of diagenetic-hydrothermal-tectonic cycles.

Turkey is located within the Mediterranean Earthquake Belt where complex rock deformation results from the continental collision

\footnotetext{
* Corresponding author.

E-mail addresses: alperbaba@iyte.edu.tr (A. Baba), hasan.sozbilir@deu.edu.tr (H. Sözbilir).
}

between the African and Eurasian plates (Bozkurt, 2001a). The border of these plates constitutes seismic belts marked by young volcanics and active faults, with the latter allowing circulation of water, as well as heat flow and geothermal energy. The distribution of hot springs in Turkey roughly parallels the distribution of the fault systems, young volcanism, and hydrothermally altered areas (Simsek et al., 2002). There are a total of about 1000 thermal and mineral water spring groups in the country (MTA, 1980; Simsek et al., 2002) (Fig. 1). This natural setting serves as a suitable environment for the presence of high levels of arsenic in geothermal fluids. The most prospective regions are the Menderes Massif, a large basement complex within the Tethyan orogenic belt that has experienced tectonic denudation and extension, accompanied by widespread hydrothermal activity, since the Miocene age. Geothermal circulation in the Menderes Massif is not driven by igneous activity, but by high thermal gradients in the extended continental lithosphere. A heat flow map of the region shows the presence of high heat flow anomalies along the grabens (Simsek, 2010a, 2010b), most likely due to magmatic intrusions. Filiz (1982) suggested that the origin of $\mathrm{CO}_{2}$ gas from the geothermal fluid along the edges of the Menderes and the Gediz Grabens is magmatic. Gulec determined that for the western part of Turkey such geothermal fluids are coming from the mantle (Gulec, 1988). Results show that meteoric waters which percolate deep into the crust gain heat from magmatic intrusions (Gokgoz et al., 2010). Although igneous activity was present during the Early Tertiary and Late Tertiary ages in 


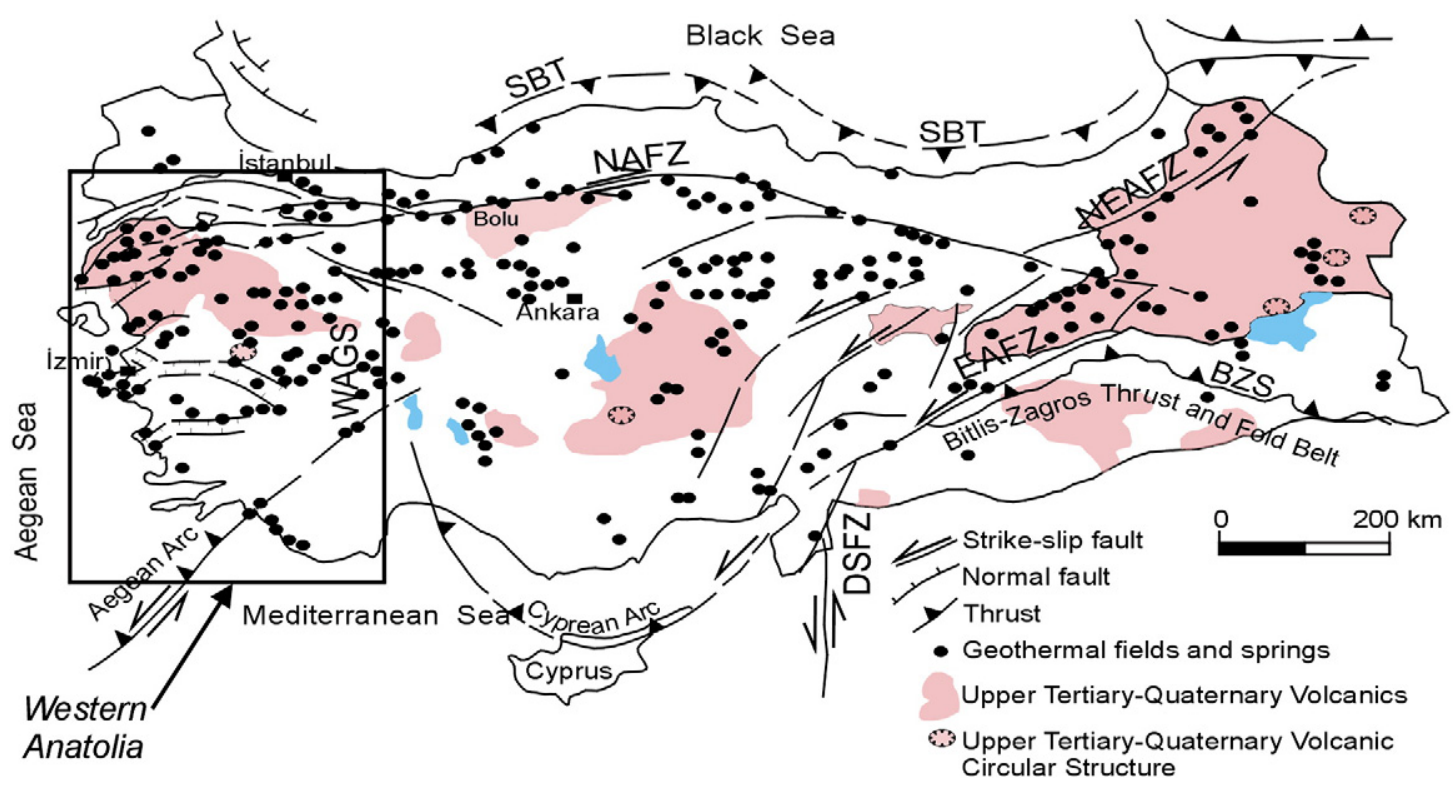

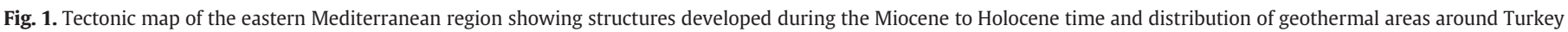

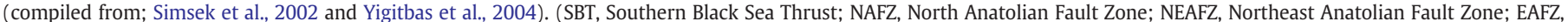
Eastern Anatolian Fault Zone; WAGS, Western Anatolian Graben System; DSF, Dead Sea Fault Zone; BZS, Bitlis-Zagros Suture) (Baba and Ármannsson, 2006).

this region, today only one small volcanic center-producing asthenospheric melts-remains in the area (Seyitoglu et al., 1997).

This study discusses the potential sources and levels of arsenic in geothermal resources of the region with particular emphasis on the sedimentological and tectonic properties of the Western Anatolian Plate.

\section{Geological setting}

Western Anatolia forms one of the most seismically active and rapidly extending regions in the world and has been currently experiencing an approximate $\mathrm{N}-\mathrm{S}$ continental extension since at least Miocene times. The $\mathrm{N}-\mathrm{S}$ extension in the region has resulted in many Neogene to Quaternary continental basins trending mainly in the E-W and NE-SW directions (Şengör et al., 1985; Yılmaz et al., 2000). The activity of the bounding high-angle normal faults is shown via numerous earthquakes (Arpat and Bingol, 1969; Şengör et al., 1985). North of the Gediz and Buyuk Menderes graben are NE-trending basins that are generally oriented at high-angles to the E-W-running basins. The best-known of these are the Gordes, Demirci, Selendi, and Usak-Gure basins (Seyitoglu et al., 1997; Bozkurt, 2003; Purvis and Robertson, 2004; Ersoy et al., 2008; Fig. 2).

Much progress have been made in the last ten years toward understanding the extensional event that affected the province (Fig. 2), especially the Gediz and the Büyük Menderes Graben and their subprovince should be classified as highly extended terranes (HET) as is the case for the Basin and Range Provinces. These terranes led to the structural juxtaposition of ductilely deformed mid-crustal rocks against brittle-deformed supracrustal rocks in an area known as the Menderes Metamorphic core complex (Bozkurt and Park, 1994), which was formed by the development of a bivergent fault system (e.g. Hetzel et al., 1995), and also containing a northerwardly dipping low-angle normal fault along the southern margin of the Gediz Graben (Hetzel et al., 1995; Emre, 1996; Emre and Sözbilir, 1997; Bozkurt, 2000; Sözbilir, 2001, 2002; Seyitoğlu et al., 2002; Yllmazer et al., 2010) and a southwardly dipping low-angle normal fault along the northern edge of the Büyük Menderes Graben (Emre and Sözbilir, 1997; Bozkurt, 2000, 2001b; Lips et al., 2001). Detachment fault systems in this province are associated with domal uplift of the Menderes metamorphic core complex, specifically of its lower plate, and the formation of asymmetric supradetachment basins in the upper plate. However, there are some studies revealing the presence of a number of NE-SW trending strike-slip faults controlling the NE-trending Miocene deposition on the western Anatolian crust onshore (Kaya, 1981; Genç et al., 2001; Kaya et al., 2004, 2007; Erkül et al., 2005; Uzel and Sözbilir, 2006, 2008; Sözbilir et al. 2008) and offshore (Ocakoğlu et al., 2004). This transversely oriented strike-slipdominated zone that accommodates the lateral termination of E$\mathrm{W}$-trending graben-faults, linking spatially the discrete loci of the terranes, includes the İzmir-Balıkesir Transfer Zone. The İzmir-Balıkesir Transfer Zone has been described as a NE-trending strike-slip dominated zone, which because of its relative inactivity limits the extensional growth of the eastern coastlines of the Aegean Sea that lie between Balıkesir and İzmir (Okay and Siyako, 1991; Ring et al., 1999; Sözbilir et al., 2003; Özkaymak and Sözbilir 2008; Uzel and Sözbilir, 2008).

Seismic tomography shows that the African slab under western Turkey is decoupled from the African Plate. This detached slab is a single, coherent body, representing the lithosphere and has been retracting since $90 \mathrm{Ma}$. There was no subduction re-initiation after slab break-off. The Anatolide-Tauride Block collision with Europe therefore did not immediately lead to slab break-off but instead to the delamination of subducting lithospheric mantle from the accreting Anatolide-Tauride Block crust, while staying attached to the African Plate. This led to asthenospheric inflow below the Anatolide-Tauride crust, high-temperature metamorphism and felsic magmatism. Slab break-off in western Turkey probably occurred $\sim 15$ Ma ago, after which an overriding plate compression and rotation accommodated ongoing Africa-Europe convergence (Hinsbergen et al., 2010).

\section{Tectonostratigraphic units in western Anatolia}

The Late Palaeozoic-Early Mesozoic units in the western Anatolian region define four distinct tectonostratigraphic units, from north to south: (i) the Sakarya Zone (e.g. the Karakaya complex) (Şengör et al., 1984; Okay and Tüysüz, 1999; Okay and Altıner, 2004), (ii) IzmirAnkara-Erzincan Suture Zone including the Karaburun Belt (Erdoğan, 1990; Okay et al., 2001; Çakmakoğlu and Bilgin, 2006) (Fig. 2), (iii) Menderes Massif-Cycladic Massif and (iv) Lycian nappes (Şengör and Yilmaz, 1981; Robertson and Pickett, 2000; Bozkurt and Oberhänsll, 2001). These units have distinct characteristics in terms of stratigraphy, 

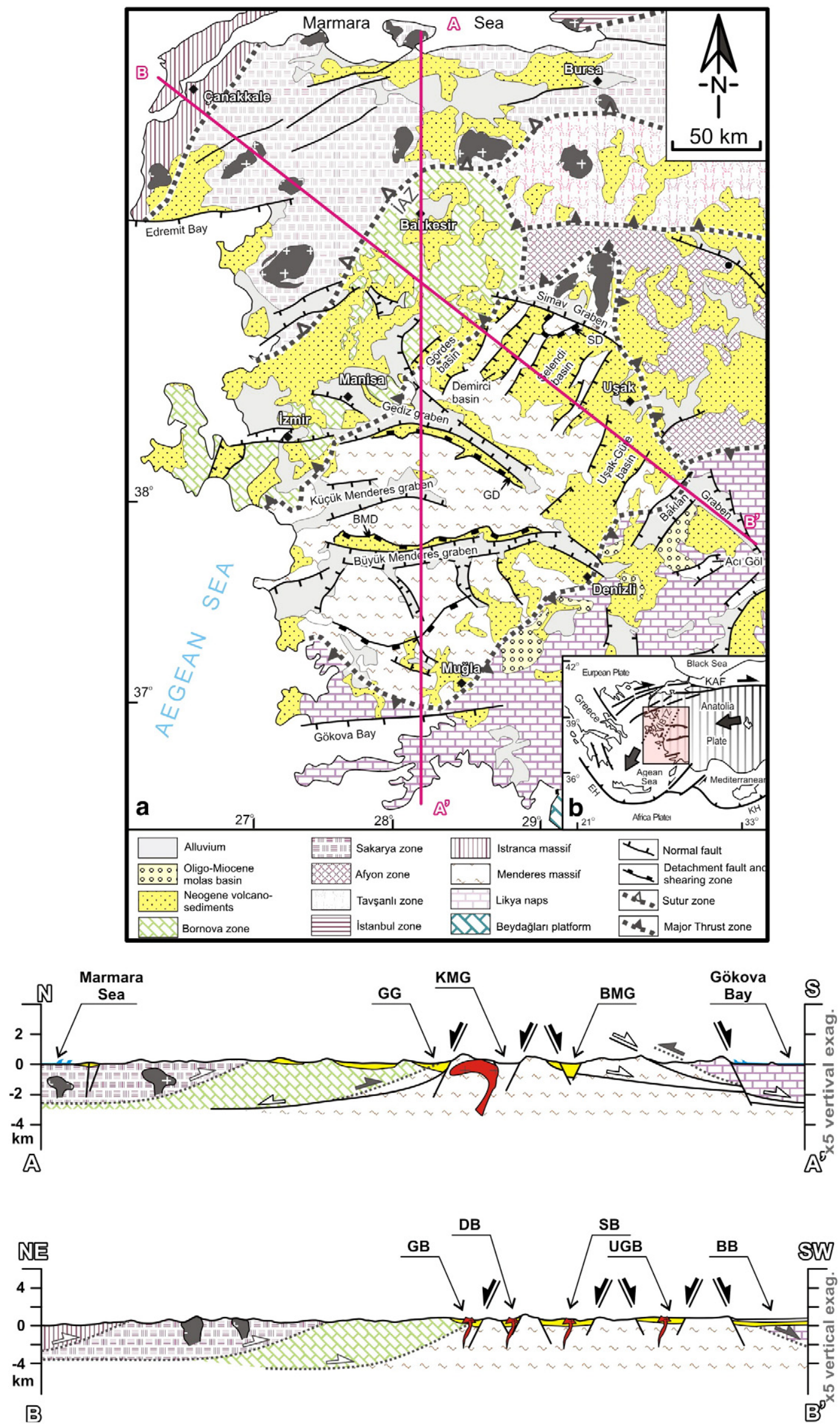

Fig. 2. Geological map and cross section of western Turkey (modified from MTA, 2002 and Sozbilir et al., 2011). 
structure, and degree of metamorphism. The Sakarya Zone consists of a highly deformed and slightly metamorphized clastic and volcanic series existing since the Permian and Triassic ages. The Izmir-Ankara-Erzincan Suture Zone is characterized by Palaeocene and younger thrust zones that form the main boundary between the Karakaya complex in the north and the Anatolide-Tauride block in the south (Erdoğan, 1990; Okay et al., 1996; Okay and Tüysüz, 1999; Okay and Altıner, 2007). The Izmir-Ankara ocean was one of the northern Neo-Tethyan strands in Anatolia. Its closure during the Palaeogenic age caused the collision between the Sakarya continent (SC) and the Taurus-Menderes platform (TMP) along the Izmir-Ankara suture zone (Şengör and Yılmaz, 1981). This collision resulted in contractional deformation during the Palaeogenic age which was succeeded by an Oligo-Miocene extension in western Anatolia (e.g. Seyitoğlu and Scott, 1996; Işı et al., 2004; Purvis and Robertson, 2004; Kaya et al., 2007).

The Karaburun Belt lies to the far west of the Izmir-Ankara-Erzincan Suture Zone with a platform-type carbonate succession, and is included in the continuation of the Sakarya continent in the classification of the cratonic realms of western Turkey (Şengör and Yllmaz, 1981). The Karaburun Belt, also known as Karaburun Mélange, is made up of Silurian-Upper Carboniferous exotic blocks within a sheared matrix of siliciclastic turbidites (Robertson and Pickett, 2000). The underlying structure of the Cycladic Massif is composed of eclogites, blueschists, and high-pressure olistostromal units (Oberhänsli et al., 2001). The Cycladic units rest on the Menderes Massif, consisting of metasediments, metabasic rocks, and Early-Middle Triassic granitic rocks (Koralay et al., 2001), and orthogneisses. Both the Cycladic and Menderes massifs are structurally overlain by metasediments that have undergone highpressure metamorphism, and ophiolites of the Lycian nappes (Oberhänsli et al., 2001; Güngör and Erdoğan, 2002). The stratigraphy of the Menderes Massif was based principally on the metamorphic sequences of the Cine submassif where a Pan-African core sequence is overlain by a Palaeozoic-Mesozoic metasedimentary cover sequence (Schuiling, 1962). However, recent studies show that this simple subdivision is incorrect (e.g. Ring et al., 1999; Gessner et al., 2001; Lips et al., 2001; Ozer and Sözbilir, 2003). According to these studies, the massif is made up of four tectonoctratigraphic units which are, from bottom to top, rudist-bearing metamorphic rocks of the Bayındır unit, a highgrade metamorphic unit intruded by Pan-African and Triassic granites, the metasedimentary Bozdag unit, the gneiss-dominated Cine unit with Pan-African granitic protoliths, and the Selimiye metasedimentary unit of the Permo-Triassic age (Gessner et al., 2001; Okay, 2001; Ozer and Sözbilir, 2003).

\section{Volcanism/magmatism of Western Anatolia}

Western Anatolia includes several different types of mineral deposits such as epithermal, porphyry, and skarn, related to the complicated tectonic and magmatic history of the region (Ozturk and Helvac1, 2008). In western Anatolia, these tectonostratigraphic units are cut by some granitoids which are sources of heat in the geothermal system of western Turkey (Fig. 2). Arsenic mobilization from rocks into the hydrosphere and pedosphere occurs in western Anatolia with mineralization and hence is a very common process in this region with volcanism and magmatisim mineralization. Mineralized rock and hydrothermal circulation, which are frequently contained in altered form are often produced by these conditions.

The genesis of Late Cenozoic volcanic and plutonic rocks throughout the Aegean Sea region was formerly ascribed to the southward migration of the Aegean subduction (e.g. Fytikas et al., 1984), but most are now considered as having resulted from regional extension and derived mainly from a hybrid magma formed by mixing of coeval mafic and felsic melts. The source of mafic magma is usually attributed to sub-continental lithospheric mantle (Seyitoğlu and Scott, 1992; Pe-Piper et al., 1995; Aldanmaz et al., 2000; Okay and Satır, 2000; Pe-Piper and Piper, 2001) or mafic lower crust (Aydogan et al., 2008;
Ozgenç and Ilbeyli, 2008). Altunkaynak and Dilek (2006) and Dilek and Altunkaynak (2007) have studied in detail the chrono-spatial evolution of the Cenozoic postcollisional magmatism in western Anatolia and concluded that the magmatism has occurred in three distinct pulses becoming younger from north to south. The first magmatic episode occurred during the Eocene and Oligo-Miocene regional compression and is attributed to an enriched subcontinental lithospheric mantle and crustal material which was also affected by an influx of asthenospheric heat and melts provided by lithospheric slab breakoff (Dilek and Altunkaynak, 2007). The second magmatic episode is ascribed to subduction-modified lithospheric mantle, crustal material and asthenospheric mantle during the Middle Miocene extensional regime as a result of delamination of the lowermost part of the lithospheric mantle, and/or partial convective removal of the subcontinental lithospheric mantle (Dilek and Altunkaynak, 2007). The third magmatic episode was generated by decompressional melting of asthenospheric mantle which commenced around $12 \mathrm{Ma}$ and continued through the Late Quaternary under the extensional tectonic regime (Dilek and Altunkaynak, 2007); the continental extension is the current tectonic regime of the western Anatolia (Bozkurt and Oberhänsl1, 2001; Emre and Sözbilir, 2007).

\section{Source for arsenic formation}

The volcanic activity that has been dominant in the geological formation of Turkey and particularly in Western Anatolia is the primary mechanism for the presence of numerous trace elements in earth's crust, including but not limited to arsenic (Baba et al., 2009c; Gunduz et al., 2010b). Arsenic levels up to $4 \%$ have been observed in mineral deposits, particularly in the Kütahya-Emet region, which is known to contain the world's largest boron deposits (Helvac1, 1986; Helvacı and Orti, 1998; Helvaci and Alonso, 2000). In the Western Anatolia region, arsenic is typically observed in the alteration zones of volcanic formations, in addition to its presence in some sedimentary rocks. Based on the tectonic characteristics (see Fig. 1) and the geological structure, many parts of Turkey are likely to have arsenic-containing geological formations within which geothermal resources are also expected to contain high arsenic levels (Table 1 ). Most of these rocks are altered and fractured, due as a result of active tectonics. Also, in Western Turkey's geothermal regions, metamorphic and sedimentary rocks are noted as being intensely hydrothermally alterated, as represented by the phyllic, argillic, and silicic-hematitic alteration zones (Ozgur et al., 1997; Baba, 2010; Gunduz et al., 2010a). Especially in some parts of western Turkey, volcanic activity led to the delineation of wide-ranging areas of alteration within mineral assemblages, from advanced argillic type to silica type to prophylitic type at deep levels. The advanced argillic alteration zones are typified by enrichments of sulfur in volcanic rocks in some

Table 1

Arsenic concentrations in rocks of Western Anatolia, Turkey (Gunduz et al., 2010a, 2010b).

\begin{tabular}{|c|c|c|c|c|}
\hline Site & Province & Type & $\begin{array}{l}\text { As } \\
(\mathrm{ppm})\end{array}$ & Reference \\
\hline Bayındır-Sarıyurt & İzmir & Ore & 200 & Bulut and Filiz (2005) \\
\hline Kızıldere & Denizli & Rock & 268 & Ozgur (2002) \\
\hline Etili & Çanakkale & Rock & 700 & $\begin{array}{l}\text { Unpublished data from Alper } \\
\text { Baba }\end{array}$ \\
\hline Çan & Çanakkale & Coal & 6413 & Baba et al. (2009a, 2009b) \\
\hline Emet & Kütahya & Rock & 3900 & Dogan and Dogan (2007) \\
\hline İğdeköy-Emet & Kütahya & Ore & 40,000 & Colak et al. (2003) \\
\hline Simav Sb Mine & Kütahya & Ore & 660 & Gunduz et al. (2010a, 2010b) \\
\hline Dulkadir & Kütahya & Rock & 4197 & Atabey (2009) \\
\hline Emet & Kütahya & Rock & 19,487 & Atabey (2009) \\
\hline $\begin{array}{l}\text { Kırka Borate } \\
\text { Mine }\end{array}$ & Kütahya & Ore & $>2000$ & Helvaci and Alonso (2000) \\
\hline Alaşehir Hg Mine & Manisa & $\begin{array}{l}\text { Waste } \\
\text { rock }\end{array}$ & 1164 & Gemici (2008) \\
\hline
\end{tabular}


parts of Western and Northwestern Turkey. Such areas are also enriched in arsenic. Oxidation of sulfide minerals, particularly of pyrite, leads to the production of ferric iron and sulfate. A similar situation can be seen for arsenopyrite (Atabey, 2009). The chemical equation of this process is given below.

$\mathrm{FeS}_{2}+7 / 2 \mathrm{O}_{2}+\mathrm{H}_{2} \mathrm{O} \rightarrow \mathrm{Fe}+2 \mathrm{SO}_{4}+2 \mathrm{H}$

$4 \mathrm{FeAsS}+13 \mathrm{O}_{2}+6 \mathrm{H}_{2} \mathrm{O} \rightarrow 4 \mathrm{Fe}+4 \mathrm{AsO}_{4}+4 \mathrm{SO}_{4}+12 \mathrm{H}$

Dogan and Dogan (2007) mention that a natural source of arsenic (As) in some parts of western Turkey comes from evaporitic minerals, carbonate, volcanic rocks, and coal. They point out that the sources of arsenic are mainly evaporitic minerals, including colemanite (2693900 ppm) and gypsum (11-99,999 ppm), but also alunite (7-10 ppm) and chert (54-219 ppm). Also they are present in secondary epithermal gypsum, which has a high concentration of As in the form of realgar and orpiment along the fracture zones of Mesozoic and Cenozoic carbonate aquifers. In addition to this they mention that limestone/dolomite (3-699 ppm) and travertine (5-4736 ppm) are relatively more enriched in As than they are in volcanics (2-14 ppm), probably because of secondary enrichment through hydrological systems.

The sulfur isotopic composition of orpiment/realgar samples of the Emet deposits in the Hisarcık open pit mine located in western Turkey shows very low $\delta^{34} S_{\text {sulfide }}$ values $(-33.7 \pm 2.3 \%$; $n=4)$. These values, which are lower than any published data for the presence of dissolved sulfide in geothermal fluids, have been attributed to a microbialmediated sulfate reduction process (Palmer et al., 2009). GarcíaVeigas et al. (2011) assume that periodic changes in redox conditions during early diagenesis led to dissolution of colloidal arsenopyrite, probably induced by micro-organisms, and to the precipitation of arsenic sulfides.

\section{Hydrogeochemical properties of geothermal resources}

Several studies were carried out about the hydrological, hydrogeochemical and geochemical features of geothermal fields in Western Turkey (Samilgil, 1966, 1985; Kasap, 1984; Simsek, 1984; Tarcan, 1995; Karamanderesi, 1997; Mützenberg 1997; Mutlu, 1998; Tarcan et al., 2000; Aksoy, 2001; Gemici and Tarcan, 2001; Tarcan and Gemici, 2001; Vengosh et al., 2002; Baba, 2003; Baba and Özcan, 2005; Baba et al., 2005; Baba and Ármannsson, 2006; Baba and Ertekin, 2007; Sanlıyüksel and Baba, 2007; Deniz et al., 2010). All these studies focus on each geothermal system. No studies focus on the potential sources and levels of arsenic in geothermal resources of the region especially with regard to particular emphasis on the sedimentological and tectonic properties of the Western Anatolian Plate. Also very few researchers measured arsenic in the geothermal fluids of Western Turkey. Most of the geothermal fluids originated from the Menderes Massif which discharges along the rims of east-west-trending faults that form the Büyük Menderes, Küçük Menderes, Gediz, and Simav grabens. Some thermal springs originate from the Kazdağı Massive in Northwestern Turkey; the circulation of the geothermal fluid is closely related to the major faults and fractured zones in these regions (Fig. 3). The Menderes Massif consists of gneisses, schists, karstic marbles, and granodiorite, including the aquifers of the geothermal systems. Also, Mesozoic carbonates are other reservoir rocks of the geothermal system in Western Turkey. Impermeable Neogene terrestrial sediments, which are made up mainly of sandy and clayey conglomerates, occur in different facies in the northern and southern parts of the grabens, since these rocks cap the geothermal systems. High concentrations of As (up to $1.42 \mathrm{mg} / \mathrm{l}$ ) can be seen in these geothermal fluids as well as a close correlation between increasing concentration and elevated temperature. In addition trace elements indicating intense, high-temperature fluid-rock interactionsuch as B (up to $67 \mathrm{mg} / \mathrm{l}$ ) and $\mathrm{Li}$ (up to $12.2 \mathrm{mg} / \mathrm{l}$ ) are found in high concentrations in geothermal fluids in western Turkey. B and Li exhibit a close correlation in the thermal waters as function of increasing temperature (Table 2).

\subsection{Biga peninsula}

The Biga peninsula is located in the northwestern part of Turkey and characterized by significant neotectonic activity. Regionally, the Biga Peninsula is closely related to the active tectonic zones of the North Anatolian Fault Zone and the West Anatolian Graben Systems (WAGS). For this reason, there are several geothermal springs with temperatures ranging from 30 to $174{ }^{\circ} \mathrm{C}$ in this peninsula. Highlevel granodiorite intrusions include the Kestanbol, Eybek, Ilıca, Kozak, Karabiga, and Evciler intrusions found here. The major geothermal regions of the Biga Peninsula are Tuzla, Kestanbol, and Hidırlar. The measured reservoir temperature of Tuzla is $174{ }^{\circ} \mathrm{C}$. The surface temperatures of Kestanbol and Hidırlar are $76{ }^{\circ} \mathrm{C}$ and $84{ }^{\circ} \mathrm{C}$, respectively. The Kocabaşlar, Kırkgeçit, Kulcüler, Palamutoba, Çan, Alibeykoy, Karaılıca, Bardakçılar, and Akçakeçili geothermal sites can be seen all around the Biga Peninsulla where the surface temperature of geothermal fluids ranges between 33 and $52{ }^{\circ} \mathrm{C}$ in springs (Table 2). Except for Tuzla and Kestanbol, the water types of this geothermal system are the $\mathrm{Na}-\mathrm{SO}_{4}$ type (Fig. 4a and b). However, Tuzla and Kestanbol water types are of the $\mathrm{Na}-\mathrm{Cl}$ type (Fig. $4 \mathrm{c}$ and $\mathrm{d}$ ). Isotopic composition studies show that the Tuzla, Kestanbol and other geothermal fluids in Biga Peninsula have different origins (Fig. 5). These results indicate that the hot saline waters (brine) in the Tuzla geothermal field originate from connate water along faults (Baba et al., 2009a, 2009b). Kestanbol water samples, depicted by oxygen $\left(\delta 1^{8} \mathrm{O}\right)$ and deuterium $\left(\delta^{2} \mathrm{H}\right)$ show that mixing of meteoric or cold water and sea water occurs. Other geothermal fluids (from Hıdırlar, Kocabaşlar, Kırkgeçit, Kulcüler, Palamutoba, Çan, Alibeykoy, Karaılıca, Bardakçılar) have the same recharge area, shallow circulation, and are meteoric in origin as depicted by their $\delta^{18} \mathrm{O}(\%)$ and $\delta^{2} \mathrm{H}(\%)$ contents (Fig. 5). Ages of these three geothermal fluids are more than 50 years old, as determined by tritium $\left({ }^{3} \mathrm{H}\right)$ data. The other geothermal systems of the Biga Peninsula show properties similar to those seen at the Hidırlar geothermal site. The highest concentration of As can be seen in Alibeyköy where the arsenic concentration ranges from $1 \mu \mathrm{g} / \mathrm{l}$ to $251.9 \mu \mathrm{g} / \mathrm{l}$ in Biga Peninsula (see Table 2).

\subsection{Gediz graben}

The Gediz graben is $140 \mathrm{~km}$ long and $3-40 \mathrm{~km}$ wide and has a WNW-ESE trending structure bounded by active normal faults. The Gediz detachment, which is located along a discontinuous trace along the fault for more than $100 \mathrm{~km}$ from Turgutlu to Alaşehir district, is one of several crustal-scale detachment faults that were formed at the edge of the southern basin of the Gediz graben (Koçyiğit et al., 1999; Sözbilir 2001, Fig. 3). The footwall of the Gediz detachment comprises mylonitic gneiss, marble, and schist of the Menderes metamorphic core complex as well as Miocene synextensional granite. The hanging wall of the detachment fault comprises Miocene to Quaternary sedimentary units reaching up to 2500 m thick.

Many geothermal resources occur along the Gediz Graben starting from the Alaşehir to the Sarıgöl Region in the east. The measured reservoir temperature has been found to be as high as $287^{\circ} \mathrm{C}$ in this graben. The major geothermal sites of the Gediz Graben are Turgutlu (Urganlı), Salihli, and Alaşehir. The measured reservoir temperatures of Salihli, Alasehir and Urganlı are $287^{\circ} \mathrm{C}, 215^{\circ} \mathrm{C}$, and $85^{\circ} \mathrm{C}$, respectively. The thermal waters of Alaşehir, Salihli, and Urganlı geothermal systems are alkaline, where carbonate alkalinity is greater than noncarbonate alkalinity (Yllmazer et al., 2010). The Kursunlu, Caferbey, Göbekli, Ufuruk, and Sart-Çamur geothermal sites are found in the Salihli Region where the surface temperature of geothermal fluid changes from 30 to $55^{\circ} \mathrm{C}$ in springs and from $51{ }^{\circ} \mathrm{C}$ to $287{ }^{\circ} \mathrm{C}$ in 

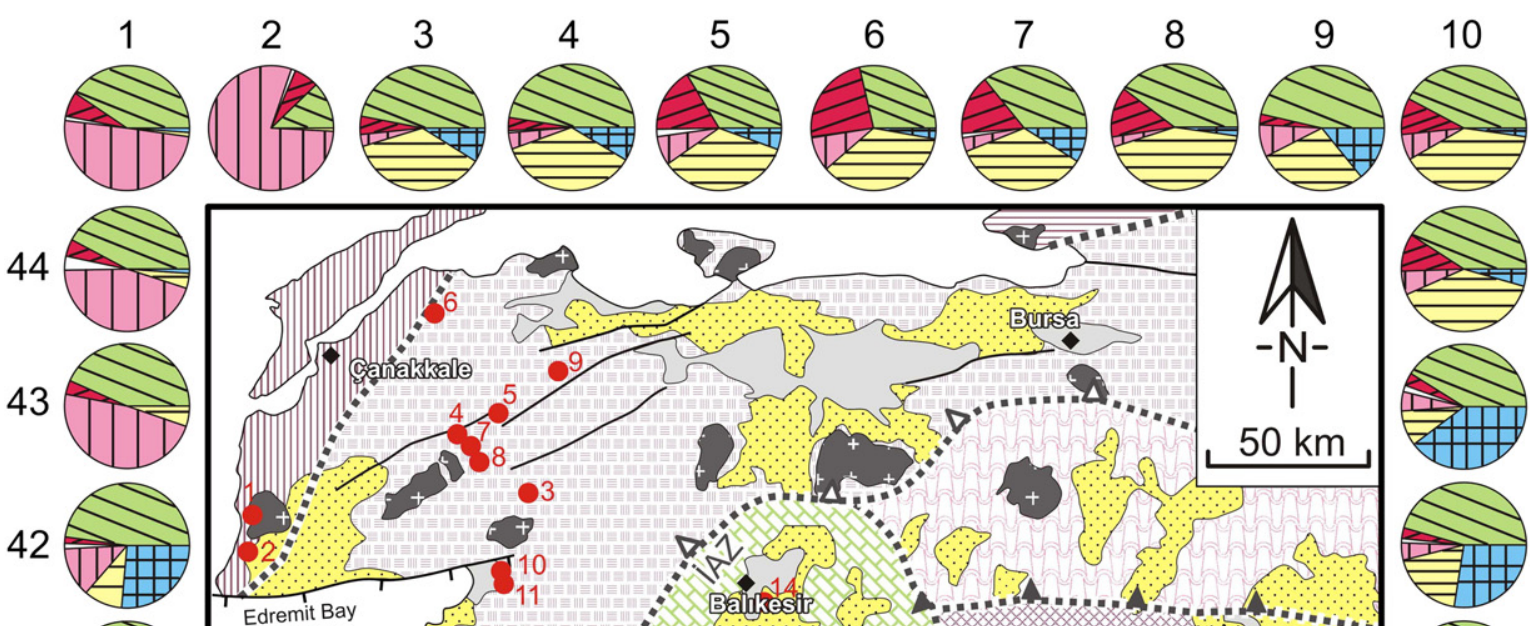

4
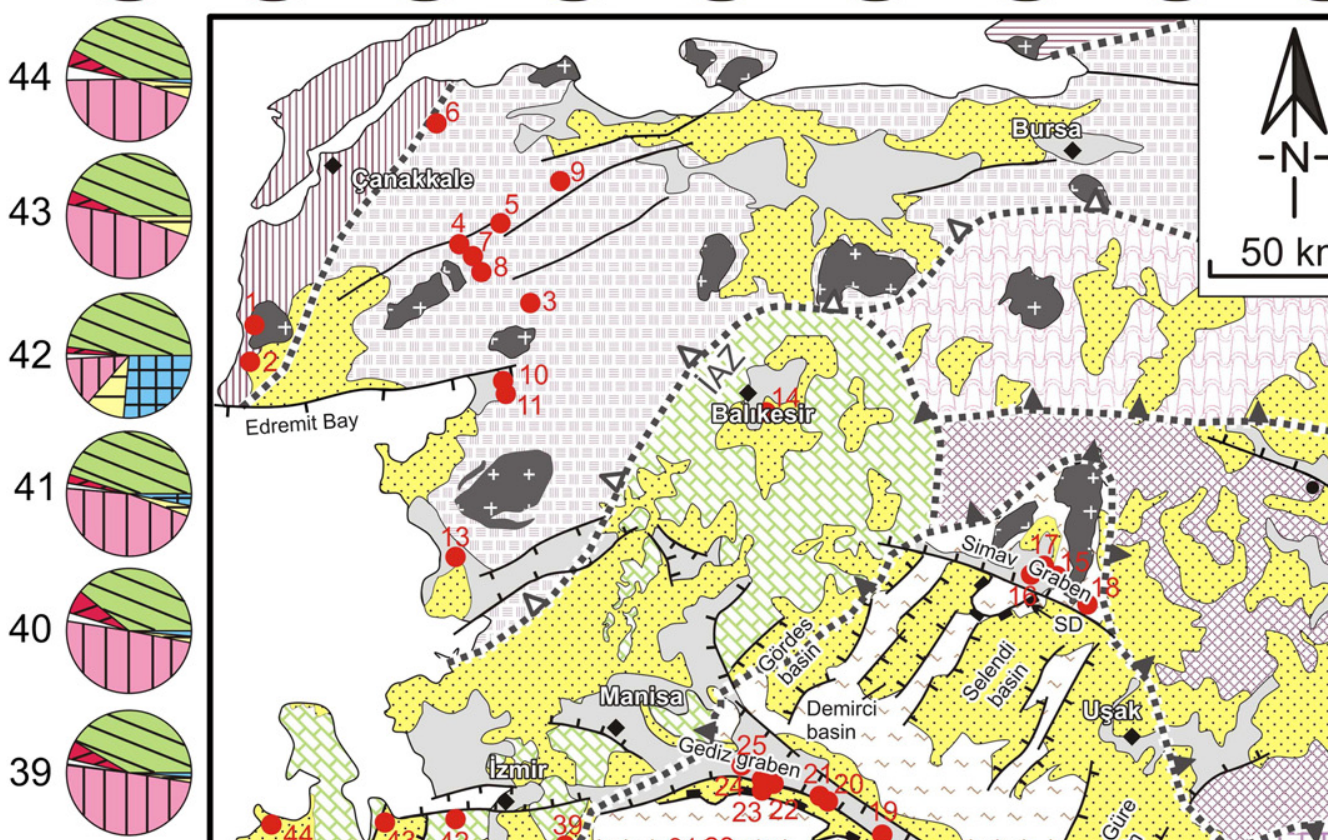

38 ग1 $38^{\circ}$
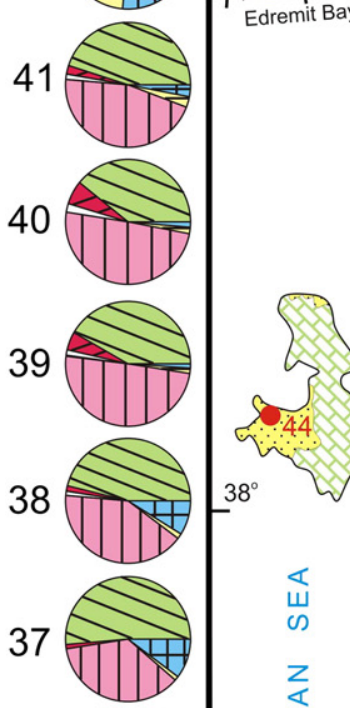

36

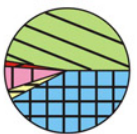

35

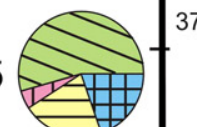

$37^{\circ}$

34

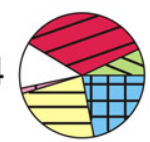

33

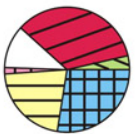

a
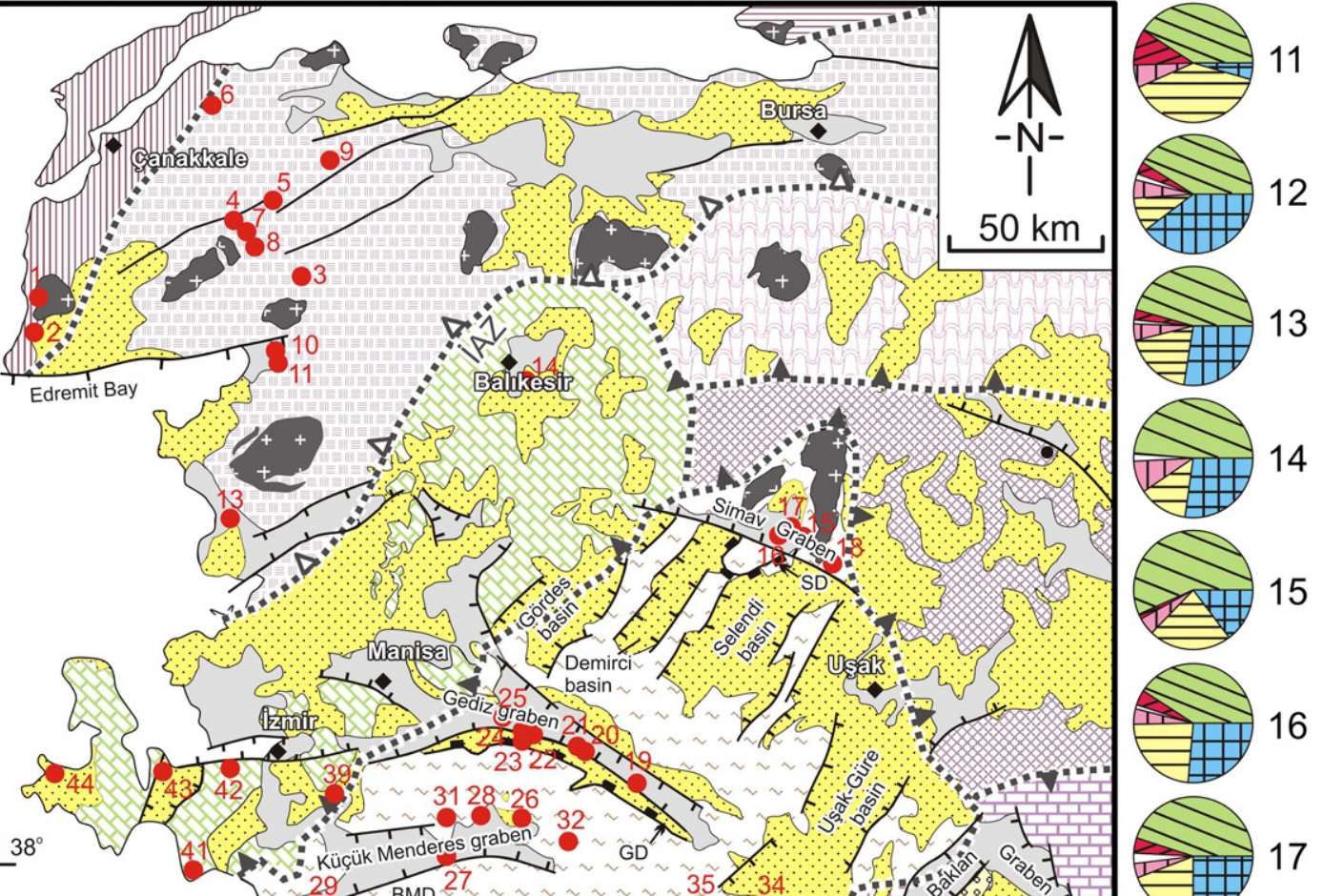

32

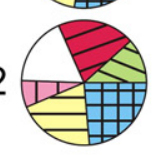

\begin{tabular}{|c|c|}
\hline & Alluvium \\
\hline 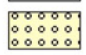 & $\begin{array}{l}\text { Oligo-Miocene } \\
\text { molas basin }\end{array}$ \\
\hline & $\begin{array}{l}\text { Neogene volcano- } \\
\text { sediments }\end{array}$ \\
\hline & Bornova zone \\
\hline
\end{tabular}

Major lons

$\triangle \mathrm{Na}+\mathrm{K}$

31

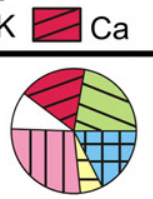

30

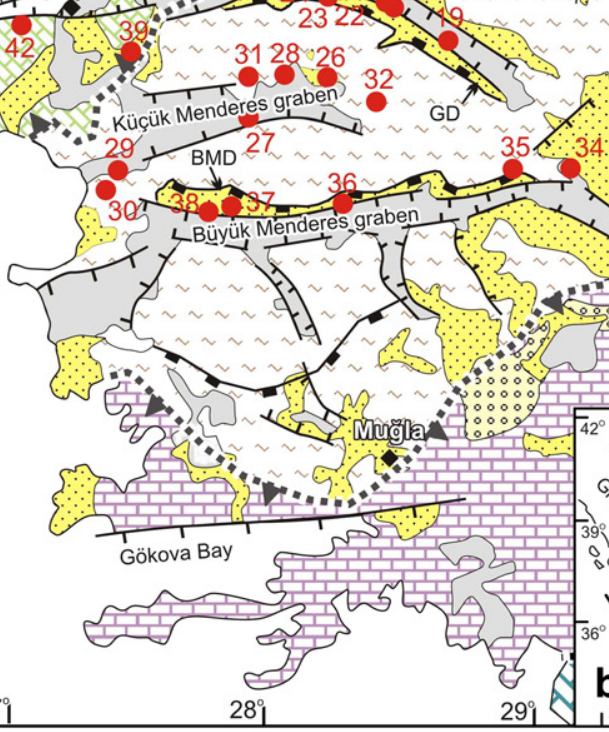

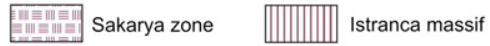

Afyon zone

$\square$ Tavşanlı zone

Elistanbul zone

$\square \mathrm{Mg}$

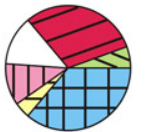

29 एवl $\quad \mathrm{SO}_{4}$

田 $\mathrm{HCO}_{3}$

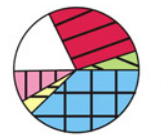

28

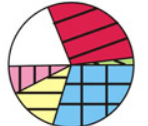

27

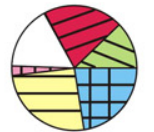

26

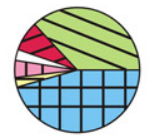

25

$50 \mathrm{~km}$
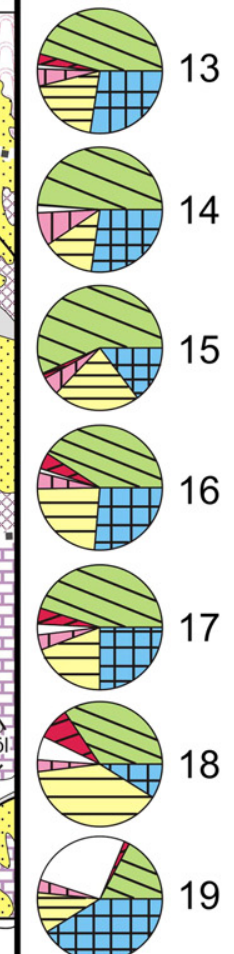

Fig. 3. General map of western Turkey and location of geothermal area.

wells. However, their discharges are between 2 and 80 l/s from either springs or wells (Ozen et al., 2010). The geothermal fluid of the Gediz Graben geothermal site has surface temperatures of $25-95{ }^{\circ} \mathrm{C}$ and has electrical conductivity of $874-6020 \mu \mathrm{S} / \mathrm{cm}$. Mineralization is mostly dominated by $\mathrm{Na}^{+}(188-2027 \mathrm{mg} / \mathrm{l})$ and $\mathrm{HCO}_{3}^{-}(544-2950 \mathrm{mg} / \mathrm{l})$ (Tarcan et al., 2005). The chemical composition of Kursunlu, Caferbey, 
Table 2

General data on geothermal systems from western Turkey.

\begin{tabular}{|c|c|c|c|c|c|c|c|c|c|c|c|c|c|c|c|c|c|}
\hline \multirow[t]{2}{*}{ Region } & \multirow[t]{2}{*}{ No } & \multirow[t]{2}{*}{ Location } & \multirow[t]{2}{*}{$\mathrm{pH}$} & \multirow{2}{*}{$\frac{\mathrm{T}}{{ }^{\circ} \mathrm{C}}$} & \multirow{2}{*}{$\frac{\mathrm{EC}}{\mu \mathrm{S} / \mathrm{cm}}$} & \multirow{2}{*}{$\frac{\mathrm{Na}}{\mathrm{mg} / \mathrm{l}}$} & \multirow{2}{*}{$\frac{\mathrm{K}}{\mathrm{mg} / \mathrm{l}}$} & \multirow{2}{*}{$\frac{\mathrm{Ca}}{\mathrm{mg} / \mathrm{l}}$} & \multirow{2}{*}{$\frac{\mathrm{Mg}}{\mathrm{mg} / \mathrm{l}}$} & \multirow{2}{*}{$\frac{\mathrm{Cl}}{\mathrm{mg} / \mathrm{l}}$} & \multirow{2}{*}{$\frac{\mathrm{SO}_{4}}{\mathrm{mg} / \mathrm{l}}$} & \multirow{2}{*}{$\frac{\mathrm{HCO}_{3}}{\mathrm{mg} / \mathrm{l}}$} & \multirow{2}{*}{$\frac{\text { As }}{\mu \mathrm{g} / \mathrm{l}}$} & \multirow{2}{*}{$\frac{B}{\mu \mathrm{g} / \mathrm{l}}$} & \multirow{2}{*}{$\frac{\mathrm{Li}}{\mu \mathrm{g} / \mathrm{l}}$} & \multirow[t]{2}{*}{ Water type } & References \\
\hline & & & & & & & & & & & & & & & & & \\
\hline Biga Peninsulla & 1 & Kestanbol & 6.4 & 74 & 3002 & 6529.6 & 759.21 & 902.3 & 65.63 & 12,929 & 112.5 & 334.2 & 184.3 & 7787 & 6367 & $\mathrm{Na}-\mathrm{Cl}$ & Baba and Deniz (2008) \\
\hline & 2 & Tuzla & 6.5 & 87 & 80300 & 5893 & 1979.2 & 2636 & 75.2 & 58,220 & 201 & 134 & 136 & 32000 & nd & $\mathrm{Na}-\mathrm{Cl}$ & Baba and Deniz (2008) \\
\hline & 3 & Hıdırlar & 7.9 & 75.5 & 1087 & 200.13 & 7.33 & 18.89 & 0.13 & 15 & 277.65 & 162 & 2.2 & 193 & 78.8 & $\mathrm{Na}-\mathrm{SO}_{4}$ & Baba and Deniz (2008) \\
\hline & 4 & Alibeyköy & 7.48 & 21 & 2460 & 612.49 & 13.91 & 38.07 & 3.84 & 83 & 970 & 290 & 251.8 & 2029 & 607.2 & $\mathrm{Na}-\mathrm{SO}_{4}$ & Baba and Deniz (2008) \\
\hline & 5 & Çan & 6.93 & 45.5 & 3160 & 606.99 & 27.42 & 259.8 & 22.12 & 201 & 1298 & 274 & 101.6 & 4050 & 1146.8 & $\mathrm{Na}-\mathrm{SO}_{4}$ & Baba and Deniz (2008) \\
\hline & 6 & Koçabaşlar & 7.4 & 31 & 1301 & 167.94 & 0.88 & 118.8 & 0.27 & 78 & 425 & 48.8 & 17.8 & 485 & 102.3 & $\mathrm{Na}-\mathrm{SO}_{4}$ & Baba and Deniz (2008) \\
\hline & 7 & Karallıca & 6.76 & 43.3 & 2470 & 489.13 & 20.89 & 183 & 7.05 & 87 & 980 & 317.2 & 55.7 & 1991 & 1082.6 & $\mathrm{Na}-\mathrm{SO}_{4}$ & Baba and Deniz (2008) \\
\hline & 8 & Bardakçılar & 8.08 & 49.1 & 1689 & 298.52 & 5.71 & 86.13 & 0.24 & 25 & 672 & 48.8 & 0.8 & 360 & 113 & $\mathrm{Na}-\mathrm{SO}_{4}$ & Baba and Deniz (2008) \\
\hline & 9 & Kırkgeçit & 8.95 & 49 & 669 & 140.56 & 1.74 & 5.88 & 0.15 & 35 & 173 & 122 & $<0.5$ & 760 & 98.6 & $\mathrm{Na}-\mathrm{SO}_{4}$ & Baba and Deniz (2008) \\
\hline Edremit Graben & 10 & Edremit & 7.83 & 57.6 & 1354 & 272.7 & 5.2 & 50.2 & 0.8 & 59.6 & 506.9 & 48.8 & 4.3 & 1190 & 305 & $\mathrm{Na}-\mathrm{SO}_{4}$ & Mutlu (2007) \\
\hline & 11 & Derman & 7.1 & 57.8 & 857 & 246 & 4.6 & 49.8 & 1.6 & 52 & 498 & 67.1 & nd & nd & nd & $\mathrm{Na}-\mathrm{SO}_{4}$ & Yalcin (2007) \\
\hline Dikili-Bergama Graben & 12 & Pasha & 6.52 & 46 & 2320 & 598 & 16.7 & 45.38 & 10.6 & 105 & 295 & 1523 & 690 & 4210 & 370 & $\mathrm{Na}-\mathrm{HCO}_{3}-\mathrm{SO}_{4}$ & Tarcan and Gemici (2010) \\
\hline & 13 & Dikili Ilıcası & 7.3 & 65 & 2900 & 660 & 42 & 37 & 7.4 & 97.7 & 564 & 1090 & nd & nd & nd & $\mathrm{Na}-\mathrm{HCO}_{3}-\mathrm{SO}_{4}$ & MTA-JICA (1987) \\
\hline Bigadiç & 14 & Hisarköy & 7.29 & 94.6 & 2820 & 706.4 & 74 & 8.4 & 11.7 & 206.7 & 381.5 & 1051.6 & 1265.5 & 8896 & 1501 & $\mathrm{Na}-\mathrm{HCO}_{3}-\mathrm{SO}_{4}$ & Mutlu (2007) \\
\hline Simav Graben & 15 & Eynal & 9.51 & 96 & 2940 & 600 & 61 & 2 & 1 & 73 & 483 & 425 & 1020 & 5400 & 1000 & $\mathrm{Na}-\mathrm{SO}_{4}-\mathrm{HCO}_{3}$ & Erisen et al. (1989), Palabiyik (2006) \\
\hline & 16 & Çitgöl & 7 & 83 & 1757 & 340 & 44 & 34 & 5.3 & 57 & 376 & 573 & 90 & 4200 & - & $\mathrm{Na}-\mathrm{HCO}_{3}-\mathrm{SO}_{4}$ & Erisen et al. (1989), Palabiyik (2006) \\
\hline & 17 & Naşa & 6.57 & 63.5 & 1621 & 395 & 42 & 39 & 9.6 & 52 & 344 & 604 & 240 & 3400 & 800 & $\mathrm{Na}-\mathrm{HCO}_{3}-\mathrm{SO}_{4}$ & Erisen et al. (1989), Palabiyik (2006) \\
\hline & 18 & Gediz & 7.3 & 74.4 & 3290 & 661.0 & 86.0 & 139.2 & 65.8 & 95.0 & 1153.5 & 1023.4 & nd & nd & nd & $\mathrm{Na}-\mathrm{SO}_{4}-\mathrm{HCO}_{3}$ & Güneș (2006) \\
\hline Gediz Graben & 19 & Alasehir & 6.82 & 30.5 & 2240 & 231.5 & 15.9 & 2.8 & 192.3 & 60 & 276 & 1477 & 5 & 18,710 & 1229 & $\mathrm{Mg}-\mathrm{Na}-\mathrm{HCO}_{3}$ & Bulbul (2009) \\
\hline & 20 & Kavaklıdere & 8.14 & 21 & 4450 & 1289.7 & 17.6 & 5 & 1.8 & 342 & 280 & 2929 & 260 & 15,001 & 643 & $\mathrm{Na}-\mathrm{HCO}_{3}$ & Bulbul (2009) \\
\hline & 21 & Göbekli & 8.31 & 95 & 6020 & 2027 & 78.9 & 12.8 & 3.1 & 165 & 1843 & 2950 & nd & nd & nd & $\mathrm{Na}-\mathrm{HCO}_{3}-\mathrm{SO}_{4}$ & Bulbul (2009) \\
\hline & 22 & Kurşunlu & 7.43 & 55 & 2780 & 539 & 60 & 35 & 11 & 98 & 58 & 1449 & nd & nd & nd & $\mathrm{Na}-\mathrm{HCO}_{3}$ & Tarcan and Gemici (2005) \\
\hline & 23 & Caferbeyli & 7.8 & 90 & 2700 & 680 & 70 & 42 & 100 & 115 & 34 & 1983 & nd & 67000 & nd & $\mathrm{Na}-\mathrm{HCO}_{3}$ & Karamanderesi (1997) \\
\hline & 24 & Sart & 6.33 & 43 & 1506 & 155.3 & 24.99 & 197.7 & 24.63 & 27 & 71.9 & 976.3 & 23.39 & 14 & 230 & $\mathrm{Ca}-\mathrm{Na}-\mathrm{HCO}_{3}$ & Ozen et al. (2010) \\
\hline & 25 & Urganlı & 6.93 & 60 & 2630 & 511 & 49 & 91 & 23 & 84 & 69 & 1679 & nd & 6000 & 1451 & $\mathrm{Na}-\mathrm{HCO}_{3}$ & Tarcan and Gemici (2005) \\
\hline Küçük Menderes Graben & 26 & Odemiş & 7.04 & 17 & 472 & 27 & 1.67 & 42 & 22 & 12 & 123 & 140 & nd & 50 & 120 & $\begin{array}{l}\mathrm{Ca}- \\
\mathrm{Mg}-\mathrm{HCO}_{3}-\mathrm{SO}_{4}\end{array}$ & Yildirim et al. (2010b) \\
\hline & 27 & Çayırlı & 7.21 & 16.8 & 869 & 11 & 2.3 & 99 & 37 & 43 & 101 & 323 & nd & 320 & 890 & $\mathrm{Ca}-\mathrm{Mg}-\mathrm{HCO}_{3}$ & Yildirim et al. (2010b) \\
\hline & 28 & Kayaköy & 7.39 & 18.5 & 468 & 11 & 0.7 & 49 & 19 & 27 & 15 & 211 & nd & nd & nd & $\mathrm{Ca}-\mathrm{mg}-\mathrm{HCO}_{3}$ & Yildirim et al. (2010b) \\
\hline & 29 & Belevi & 7.3 & 19.7 & 890 & 21 & 2.9 & 108 & 27 & 65 & 34 & 375 & nd & 80 & 370 & $\mathrm{Ca}-\mathrm{HCO}_{3}$ & Yildirim et al. (2010b) \\
\hline & 39 & Selçuk & 7.4 & 18.2 & 1615 & 153 & 12.5 & 100 & 38 & 290 & 77 & 328 & nd & 100 & 970 & $\mathrm{Na}-\mathrm{Ca}-\mathrm{HCO}_{3}$ & Yildirim et al. (2010b) \\
\hline & 31 & Bayındır & 7 & 38 & 1231 & 159 & 13.8 & 61 & 8 & 21 & 29 & 597 & nd & 700 & 400 & $\mathrm{Na}-\mathrm{HCO}_{3}$ & Yildirim et al. (2010b) \\
\hline & 32 & Beydağı & 7.32 & 18 & 907 & 53 & 3.9 & 77 & 44 & 48 & 185 & 280 & nd & 100 & 650 & $\begin{array}{l}\mathrm{Ca}- \\
\mathrm{Mg}-\mathrm{HCO}_{3}-\mathrm{SO}_{4}\end{array}$ & Yildirim et al. (2010b) \\
\hline Büyük Menderes Graben & 33 & Pamukkale & 6.68 & 33 & 2520 & 40 & 5.2 & 450 & 86 & 12.2 & 610.1 & 1049 & nd & nd & nd & $\mathrm{Ca}-\mathrm{Mg}-\mathrm{HCO}_{3}$ & Dilsiz (2006), Tokçaer (2007) \\
\hline & 34 & Karahayit & 7 & 60 & 2900 & 116.8 & 23.6 & 505.2 & 117.2 & 24.5 & 863 & 964 & 7 & 120 & nd & $\mathrm{Ca}-\mathrm{Mg}-\mathrm{HCO}_{3}$ & Dilsiz (2006), Gokgoz et al. (2010) \\
\hline & 35 & Kızıldere & 8.9 & 102 & 5080 & 1337 & 147 & 1.5 & 0.2 & 156 & 964 & 1306 & 1080 & nd & nd & $\mathrm{Na}-\mathrm{HCO}_{3}$ & Ozgur (2002) \\
\hline & 36 & Salavatlı & & 167 & & 1260 & 105 & 6 & 1 & 250 & 150 & 2900 & nd & 51.100 & nd & $\mathrm{Na}-\mathrm{HCO}_{3}$ & Vengosh et al. (2002), \\
\hline & 37 & Germencik & 8.7 & 231 & & 1600 & 145 & 6 & 1.2 & 1700 & 24 & 900 & nd & nd & nd & $\mathrm{Na}-\mathrm{Cl}$ & Gemici and Tarcan (2001); Simsek (2003) \\
\hline & 38 & Gümüşköy & 8.2 & 80 & 11,560 & 2650 & 208 & 32 & 11 & 3456 & 70 & 1482 & nd & nd & nd & $\mathrm{Na}-\mathrm{Cl}$ & Simsek (2003), Yildirim et al. (2010a) \\
\hline Seferihisar & 39 & Cumalı & 6.25 & 66 & 30200 & 6058 & 1034 & 651 & 71 & 11,050 & 363 & 469 & 172 & 16,600 & 12,230 & $\mathrm{Na}-\mathrm{Cl}$ & Conrad et al. (1995), Tarcan and Gemici (2003) \\
\hline & 40 & Tuzla spring & 6.28 & 65 & 34,200 & 6328 & 759 & 794 & 225 & 12,200 & 602 & 466 & 75 & 12,800 & 9550 & $\mathrm{Na}-\mathrm{Cl}$ & Conrad et al. (1995), Tarcan and Gemici (2003) \\
\hline & 41 & Doğanbey & 6.9 & 72 & 7515 & 2750 & 130 & 128 & 38 & 4320 & 332 & 515 & 78 & 9800 & 5030 & $\mathrm{Na}-\mathrm{Cl}$ & Tarcan and Gemici (2003). \\
\hline & 42 & Balçova & 7.77 & 129 & 1842 & 459.5 & 36.5 & 13.22 & 2.19 & 200 & 186 & 664 & 1419 & 21,333 & 1873 & $\mathrm{Na}-\mathrm{HCO}_{3}-\mathrm{Cl}$ & $\begin{array}{l}\text { Aksoy et al. (2009), Simsek (2010a, 2010b), } \\
\text { Tarcan and Gemici (2010) }\end{array}$ \\
\hline & 43 & Urla & 7.39 & 37 & 34,390 & 11468 & 469 & 1383 & 835 & 19,877 & 2865 & 140 & 24 & 4237 & 333 & $\mathrm{Na}-\mathrm{Cl}$ & Tarcan (2000) \\
\hline & 44 & Çeşme & 6.5 & 58 & 26,600 & 7108 & 631 & 677 & 367 & 11,530 & 1665 & 195 & & & & $\mathrm{Na}-\mathrm{Cl}$ & Gemici and Filiz (2001) \\
\hline
\end{tabular}


a

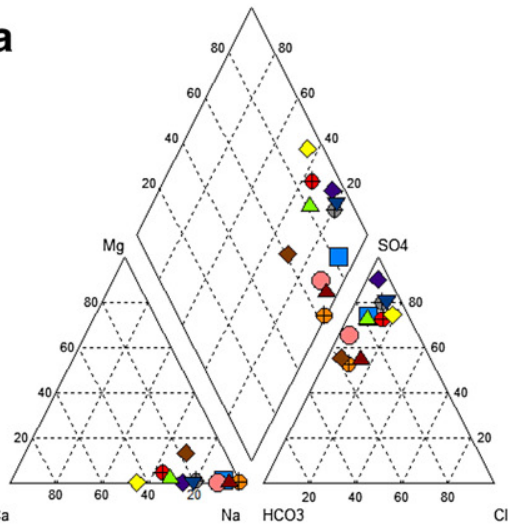

C

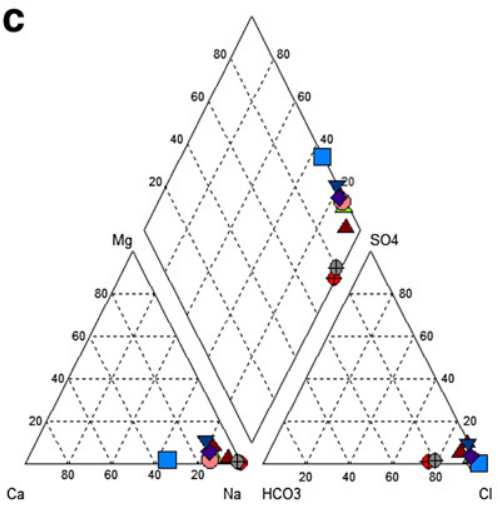

e

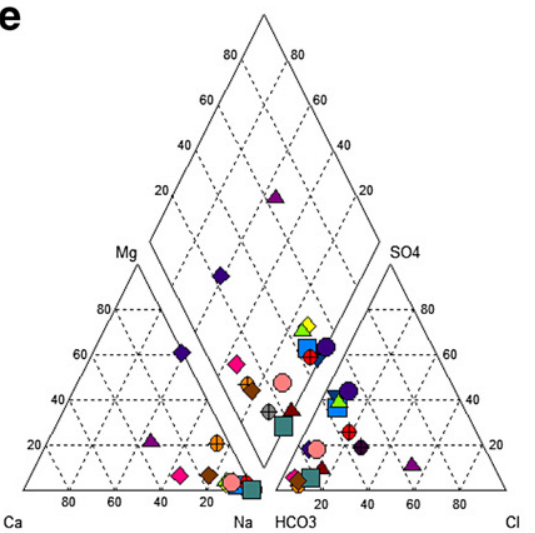

g

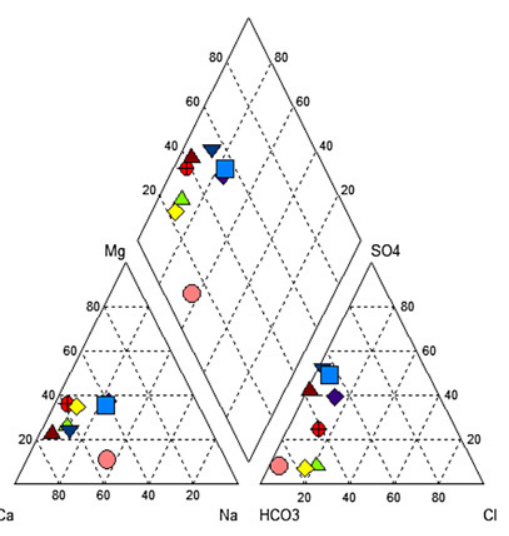

b

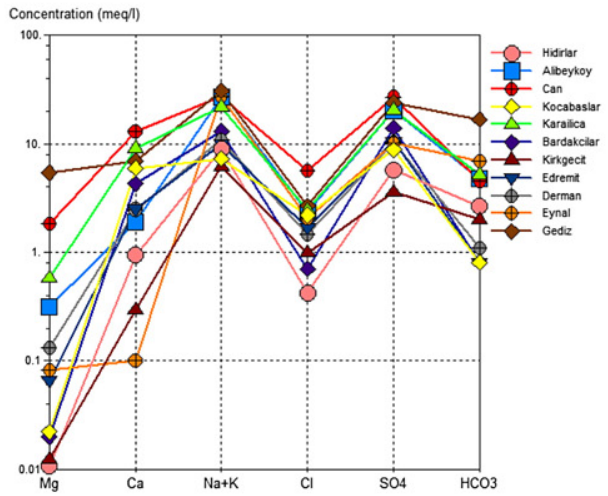

d

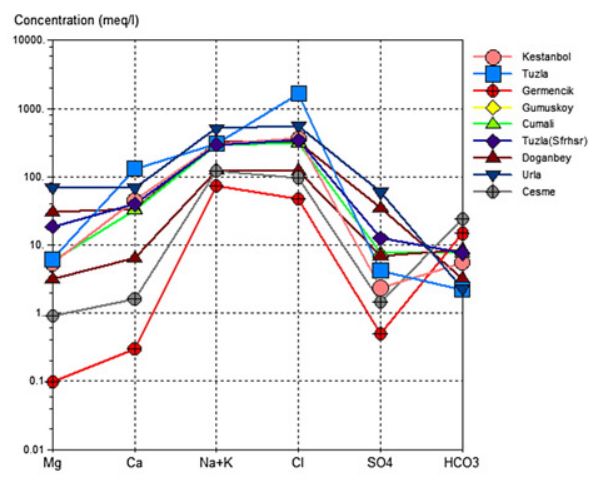

f

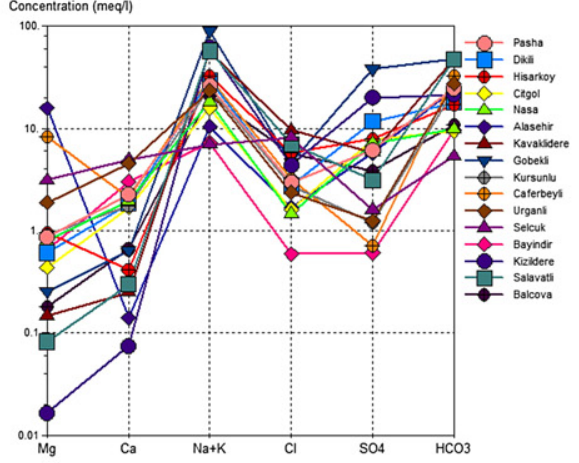

h

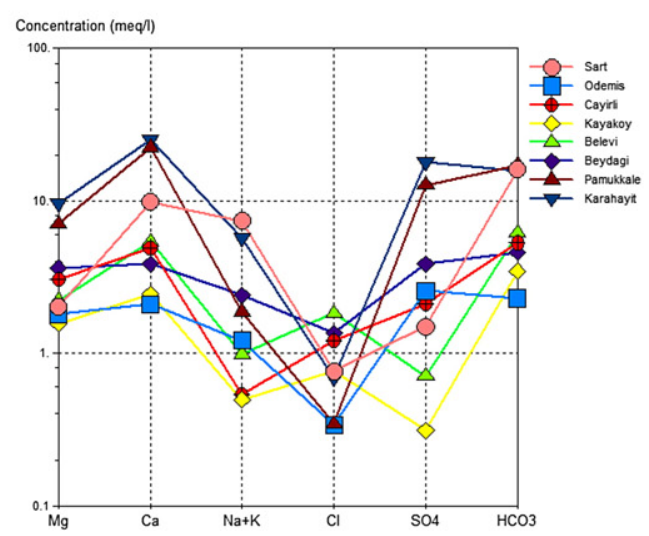

Fig. 4. Chemical analysis of water in the study area plotted on Piper and Schoeller diagrams. 

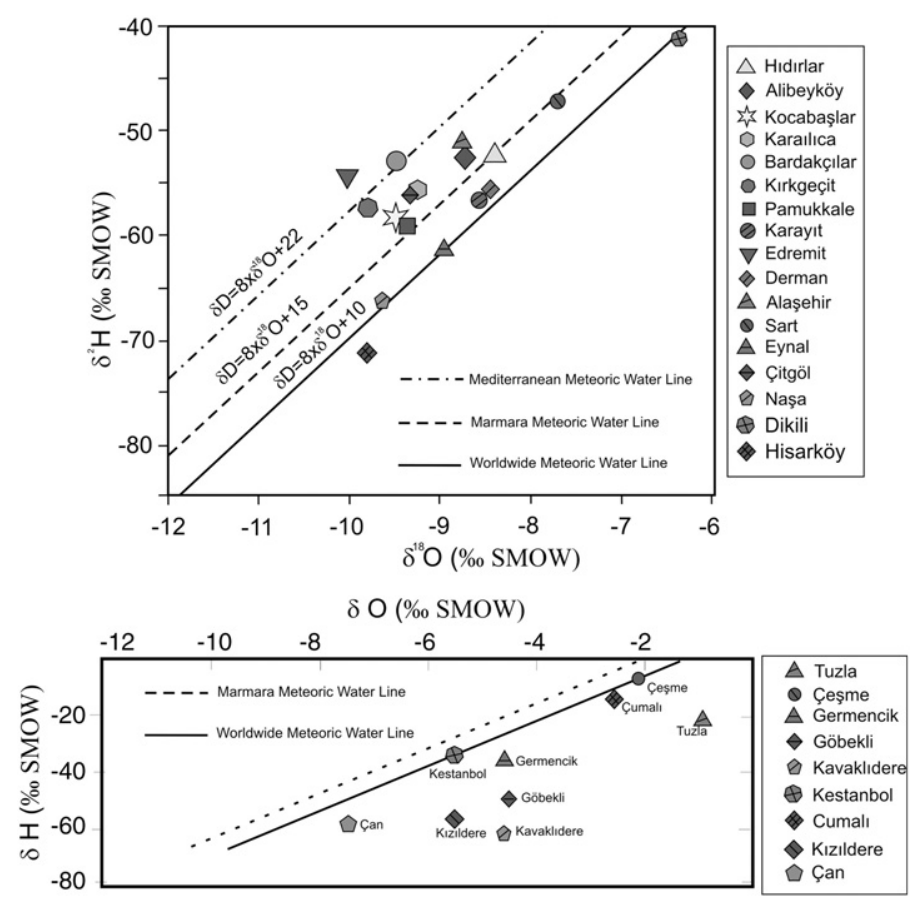

Fig. 5. Stable isotope compositions of the geothermal reservoir fluids in western Turkey.

and Sart-Camur is of the $\mathrm{Na}-\mathrm{HCO}_{3}, \mathrm{Na}-\mathrm{HCO}_{3}$ (Fig. 4e and f), and Ca$\mathrm{Na}-\mathrm{HCO}_{3}$ types (Fig. $4 \mathrm{~g}$ and $\mathrm{h}$ ), respectively.

According to isotopic $\left(\delta^{18} \mathrm{O}-\delta^{2} \mathrm{H}\right)$ results, the geothermal fluid from Gediz Graben is fed by meteoric waters (Tarcan and Filiz, 1997) (Fig. 5). The Sart-Çamur Geothermal field is located in the southeastern part of the ancient city of Sardes, located in the Gediz region on the Kavaklidere River, where the highest As values $(260 \mu \mathrm{g} / \mathrm{l})$ were measured in the thermal spring (Ozen et al., 2010). These thermal springs are located on the detachment fault of the Gediz Graben.

\subsection{Buyuk menderes graben}

Buyuk Menderes Graben contains the valley of the Buyuk Menderes River, which is $150 \mathrm{~km}$ long and $10-20 \mathrm{~km}$ wide. The graben is bordered by well-developed normal fault systems along its length. Rock units exposed in the vicinity of Buyuk Menderes Graben can be classified into two groups as the basement and basin fill units. Metamorphic rocks belonging to the Menderes Massif constitute the pre-Neogene basement units, which is an extensional metamorphic core complex in the Western Anatolian extensional province (Bozkurt, 2001b). The basin fill consists of four sedimentary packages that formed on the metamorphic rocks of the Menderes Massif (e.g. Sözbilir and Emre, 1990; Bozkurt, 2000). These are, from bottom to top, the early-middle Miocene Haskoy Formation, late Miocene Gokkırantepe formation, late Pliocene-Pleistocene Asartepe formation and Quaternary alluvium (Sözbilir and Emre, 1990). Detailed descriptions of the sequence stratigraphic units are given in Sözbilir and Emre (1990), Seyitoğlu and Scott (1992), Cohen et al. (1995) and Bozkurt (2000).

Büyük Menderes Graben is one of the most developed regions in Western Anatolia for geothermal energy research. The geothermal system is controlled by the active graben faults in the region. The reservoir rocks in the geothermal field are limestone in a Neogene complex, while marbles and quartzite schist exist in the Paleozoic basement complex. Most of the important geothermal systems can be seen between the Denizli and Aydın regions. Kızıldere, Gölemezli, and Yenice located in Denizli. Germencik, Ömerbeyli, Salavatli, Yilmazköy, Imamköy, and Ilıcabasi are located in the Aydın region. These geothermal sites have medium and high enthalpy fluid. The first electric power plant in Turkey, having an output capacity of 20.4 MWe was constructed in the Kızıldere (Denizli) geothermal site $\left(200-242{ }^{\circ} \mathrm{C}\right)$. Turkey has six electric power plants that produce a total output capacity of 100 MWe via geothermal sources. Research on geothermal energy has been carried out at the Germencık-Aydın $\left(200-232{ }^{\circ} \mathrm{C}\right)$, Sultanhisar-Aydın $\left(145^{\circ} \mathrm{C}\right)$ Salavatli-Aydın $\left(171{ }^{\circ} \mathrm{C}\right)$, Yilmazköy-Aydın $\left(142{ }^{\circ} \mathrm{C}\right)$, Atca-Aydın $\left(124^{\circ} \mathrm{C}\right)$, Nazili-Aydın $\left(155^{\circ} \mathrm{C}\right)$, Umurlu-Aydın $\left(155^{\circ} \mathrm{C}\right)$, and Ilıcabasi-Aydın $\left(101^{\circ} \mathrm{C}\right)$, Gölemezli $\left(75-88^{\circ} \mathrm{C}\right)$ and Yenice $\left(36-63^{\circ} \mathrm{C}\right)$ sites in Denizli. Other important geothermal fields are the Tekkehamam, Buldan, Karakova, and Karahayit-Pamukkale sites in the Denizli region where the geothermal fields are located along the edges of the Menderes and Gediz Graben and the Germencik Camur-Bozköy-Alangüllü, Nazilli Basin (Güvendik-Gedik-Nazilli), and Söke fields in the Aydın region (Simsek, 2003). The results of analyses show that geothermal waters in the Aydin region change from site to site. Examples of high temperature wells of Gümüşköy are characterized by higher mineralization with the predominant ions being $\mathrm{Na}^{+}, \mathrm{K}^{+}$and $\mathrm{Cl}^{-}$, and $\mathrm{HCO}_{3}^{-}$. On the other hand, the mineralization of the "low temperature" springs of Argavlı and Sazlıköy is much lower and dominated by the presence of $\mathrm{Ca}^{2+}, \mathrm{Mg}^{2+}$, and $\mathrm{HCO}_{3}^{-}$. The natural artesian discharge of Gümüşköy shallow mixture water displays intermediate mineralization between the two main groups, both of which are of the NaCl-type (Yildirim et al., 2010a). Also Germencik geothermal mixture water is mainly of the $\mathrm{NaCl}$ type (Fig. $4 \mathrm{c}$ and $\mathrm{d}$ ) and of the $\mathrm{Na}-\mathrm{HCO}_{3}$ type (Fig. 4e and f) in the Salavatlı site. Thermal waters from Pamukkale springs are dominated by $\mathrm{Ca}-\mathrm{Mg}-\mathrm{HCO}_{3}$ (Fig. $4 \mathrm{~g}$ and $\mathrm{h}$ ). The Karahayit geothermal site is located at the intersection of the Buyuk Menderes and Gediz grabens in the Denizli Province of Western Turkey. The reservoir rocks at the site are Paleozoic marble, various schist, and quartzite, all of which have high secondary permeability. The impermeable sediments of Neogene have good cap rock characteristics. The temperatures of thermal waters at the site range from 33 to $61.5^{\circ} \mathrm{C}$. The thermal waters are of the $\mathrm{Ca}$ $\mathrm{SO}_{4}-\mathrm{HCO}_{3}$ type and of meteoric origin (Gokgoz et al., 2010). The chemistry of these thermal waters is probably dominated by a combination of mixing with cold waters, mineral dissolution-saturation reactions, and ion exchange reactions. Ion concentrations of hot waters from deep wells are higher than those of hot springs. However, 
their minor constituents are variable. Arsenic concentrations $(1080 \mu \mathrm{g} / \mathrm{l})$ of thermal waters at the Kizıldere geothermal sites are high. The tritium contents of the geothermal waters in Kizıldere, Aydın, and Germencik indicate that the residence time of recharging water in the geothermal system is more than 50 years while at the Pamukkale and Söke sites the thermal waters appear to be younger. There is a clear $\delta^{18} \mathrm{O}$ shift from MMWL in the Kizıldere, Aydın, and Germencik high temperature fields (Simsek, 2003) (Fig. 5).

\subsection{Küçük menderes graben}

The Küçük Menderes Graben (KMG) is part of the horst-graben system of Southwestern Anatolia, Turkey. It is bounded by the Bozdağ horst in the north and by the Aydin horst in the south. The E$\mathrm{W}$-trending KMG is $80-\mathrm{km}$ long and $3-10 \mathrm{~km}$ wide. It was developed over the pre-Miocene basement rocks comprising metamorphic rocks of the Menderes Masif, cut by the Miocene andezitic, basaltic rocks, and the Upper Cretaceous-Palaeocene Bornova melange. It is filled with Miocene-Quaternary continental deposits. The Miocene-Quaternary sequences are composed of five distinct rock types, from bottom to top: Miocene-Pliocene continental deposits, Pliocene fluvial clastics, Plio(?)Quaternary elevated fluvial clastics, Quaternary alluvial fans and Quaternary alluvium. The Miocene-Pliocene continental deposits are widespread to the north of Torbalı and west of Selçuk. Other limited examples are seen in the northeast of Kiraz, as well as in the east and west of Tire.

Many springs can be seen in the Küçük Menderes Graben starting from towns of Ödemiş to Selçuk in the west. But most of these springs have low temperatures. The highest temperature $\left(48^{\circ} \mathrm{C}\right)$ of these geothermal fluids was measured in Bayındır, near Ödemiş. Except for the Bayındır spring, Çayırlı, Kayaköy, Beydağ and Ödemiş water samples fall into the earth-alkaline (Ca-Mg)-bicarbonate waters category, which is linked to shallow circulation. The Bayındır spring is of the $\mathrm{Na}-\mathrm{HCO}_{3}$ type (Fig. 4e and f). There have not been any analyses performed concerning arsenic and isotopes in the water resources of the Küçük Menderes Graben.

\subsection{Seferihisar region}

The Seferihisar region is located at the western termination of the Küçük Menderes Graben, close to the southeastern part of the İzmirBalıkesir Transfer zone. The stratigraphy of the study area is considered within three main groups: (1) the basement rock units, which consist of Palaeozoic-Mesozoic metamorphic rocks of the Menderes Massif and the Upper Cretaceous-Paleocene rocks of the Bornova mélange; (2) Neogene volcano-sedimentary units (ancient basin fill units); and (3) Plio-Quaternary units (modern basin fill units). The metamorphic rocks of the Menderes Massif consist of schists and marbles with local phyllite intercalations (Güngör and Erdoğan, 2002). The Bornova mélange is made up of a deformed and locally metamorphized flysch-like matrix from the Maastrichtian-Paleocene age in which blocks of Mesozoic limestones, serpentinites, and submarine volcanics occur (Erdoğan, 1990). The Menderes Massif and tectonically overlying Bornova mélange are unconformably overlain by the Neogene volcano-sedimantary successions.

The Seferihisar Geothermal system is located south of the city of Izmir where several geothermal sites have been investigated since 1970. This geothermal system is characterized by an average heat flow of $110 \mathrm{~mW} \mathrm{~m}^{-2}$ (Ilkisik, 1995; Sari and Salk, 2003) and abundant geothermal activity controlled by strike-slip faults. The strike-slip and normal faults break into several splays along which natural hot springs form (Magri et al., 2010). The $\mathrm{Na}-\mathrm{Cl}$ thermal waters of Seferihisar have measured surface temperatures of $66-72{ }^{\circ} \mathrm{C}$. The reservoir temperature of this region reaches $153{ }^{\circ} \mathrm{C}$. Bornova melange rocks, which consist of sandstone shale intercalations, conglomerate, mafic submarine volcanics, limestone lenses, serpentinite and limestone bodies, and their complexes, through the intersection of faults in this region. The clayrich zones of the overlying Neogene terrestrial sediments cap the system. The heat source is the high geothermal gradient caused by the graben tectonics of the area. $\mathrm{Na}^{+}$and $\mathrm{Cl}^{-}$ions mainly dominate the chemistry of these thermal waters, thus the thermal waters of the Seferihisar area appear to be mixtures of groundwaters and seawater (Tarcan and Gemici, 2003). The concentrations of As range from 78 to $172 \mu \mathrm{g} / \mathrm{l}$ in the Seferihisar geothermal field (see Table 2).

\section{6. İzmir bay region}

İzmir Bay is a typical depression for the Aegean back-arc domain undergoing $\mathrm{N}-\mathrm{S}$ extension which is accommodated by active dip- to oblique-slip normal faults and strike-slip faults. The major part of the modern depression is flooded by the waters of Aegean Sea, forming the bay of İzmir.

The southern edge of the bay is characterized by the Balçova geothermal system, which is one of the most important geothermal locations for the city of İzmir, and supplies hot water to the Balçova district heating system. The geothermal fluid of this region is rich in terms of $\mathrm{Na}^{+}$and $\mathrm{HCO}_{3}^{-}$. The Balçova geothermal sites are fed by meteoric groundwater seeping into the reservoir unit from terrains at elevations of about $500 \mathrm{~m}$ above sea level (Yllmazer, 1989; Aksoy, 2001; Aksoy et al., 2009). The highest concentration of arsenic $(1419.8 \mu \mathrm{g} / \mathrm{l})$ was measured in the Balçova district geothermal fluid (see Table 2).

\subsection{Simav graben}

Simav graben is a E-W trending Pliocene to Quaternary asymmetric depression that developed on the older NE-SW trending Miocene basins in Western Anatolia (Seyitoğlu, 1997). The graben is bounded from the south by an active oblique-slip normal fault, the Simav fault. The Graben fill associated with the Simav fault is composed of semlithified boulder conglomerate and sandstone. The northern part of the Simav graben is known as the Akdere basin, which consists of coarse clastics and Naşa volcanics.

The Simav geothermal field, one of the most important geothermal fields, is located in Kütahya's Simav graben system of Western Anatolia. The geothermal fluid of this region is rich in terms of $\mathrm{Na}-\mathrm{HCO}_{3}-\mathrm{SO}_{4}$ (Fig. 4a and b) and $\mathrm{Na}-\mathrm{SO}_{4}-\mathrm{HCO}_{3}$ (Fig. 4e and f) and is affected by groundwater which is low in the concentration of $\mathrm{Cl}^{-}$. The water is of meteoric origin and belongs mostly to the immature water group (Palabiyik and Serpen, 2008). The concentration of As ranges from 90 to $1020 \mu \mathrm{g} / \mathrm{l}$ in the Simav geothermal site. The highest concentration of arsenic was measured in Eynal geothermal fluid (Palabiyik and Serpen, 2008).

\subsection{Edremit graben}

The Edremit graben which is $80 \mathrm{~km}$ long and $5 \mathrm{~km}$ wide, is an offshore graben of Western Anatolia. The northern margin of the graben is bounded by the Kazdağ Mountain, which rises from sea level to over $1000 \mathrm{~m}$. The major faults bounding the Edremit gulf forms western branches of the North Anatolian fault Zone (Yllmaz et al., 2000). The most prominent topographic feature of the region is the Kazdağ 1 horst formed in the late Paleozoic-Triassic period with exposed metamorphic rocks. The late Oliocene-Early Miocene Karaköy, Evciler and Kestanbol granodioritic plutons are intruded into the metamorphic basement. This unit is overlain by Miocene volcanosedimentary units (Siyako et al., 1989). Many geothermal springs have been seen in this graben. The surface temperature of these springs changes from 55 to $65{ }^{\circ} \mathrm{C}$. The geothermal fluid of this region is rich in terms of $\mathrm{Na}-\mathrm{SO}_{4}$ as a result of the fault. The geothermal fluids of this region do not contain arsenic. 


\subsection{Dikili-bergama graben}

The Bergama graben is one of the major depressions of western Anatolia. It is a V-shaped depression trending NE-SW along the Bergama district and NW-SE along the Dikili district. The faults along both sides of the grabens indicate oblique-slip normal faults.

The Kozak horst consisting of metamorphic (Triassic Karakaya formation), plutonic (latest Oligocene-Early Miocene Kozak granite) and volkanosedimentary units, rises to over $1000 \mathrm{~m}$ with respect to the Bergama and Dikili grabens. The region is mainly delineated by two sets of oblique-slip faults trending in the NE-SW and NW-SE directions (Karacik et al., 2007). All the products of the calc-alkaline Miocene volcanism of Western Anatolia crop out in this area. The Dikili-Candarlı high consists essentially of volcanic rocks. Two major rock groups have been distinguished: the Dikili and the Candarlı groups (Karacık and Yılmaz, 2000). The Dikili group is Early-Middle Miocene in age and consists mainly of pyroclastic rocks, lavas, and associated sedimentary rocks. The Candarlı group consists of Upper Miocene-Pliocene sediment association and volcanic rocks, which are rhyolitic domes and basaltic andesite-basalt lavas-dikes. The contact between Dikili and Candarlı groups is an unconformity surface.

The Dikili Bergama geothermal site is one of the most important geothermal sites, and is located in the north of Izmir. Many geothermal springs are exposed in this region. Geothermal fluids of this region are rich in terms of $\mathrm{Na}-\mathrm{HCO}_{3}-\mathrm{SO}_{4}$ as a result of faults. The water is of meteoric origin. The highest concentration of arsenic for this region $(690 \mu \mathrm{g} / \mathrm{l})$ was measured in the Pasha geothermal fluid.

\subsection{Karaburun Peninsula}

The Neogene stratigraphy of the Karaburun Peninsula is represented by a volcano-sedimentary succession, including several sedimentary and volcanic units. These units rest on a basement comprising nonmetamorphic and intensely sheared Paleozoic to Mesozoic rocks of the Karaburun belt (e.g., Erdoğan, 1990; Robertson and Pickett, 2000; Tatar-Erkül et al., 2008). The Neogene volcano-sedimentary units begin at the Bozköy Formation consisting of conglomerates of alluvial fans of fluvial origin. The unit laterally and vertically passes into the Urla limestone that is composed of mainly white-colored fresh-water limestones. In the Karaburun Peninsula, several volcanic units interfinger with the Urla limestone. These are Karaburun, Armağandağ, Kocadağ, and Yaylaköy volcanics, which were emplaced contemporaneously with deposition of the Urla limestone during latest early Miocene. These units are unconformably overlain by the Urla volcanics and finally the Ovacik basalt. The Urla volcanics have been dated by Borsi et al. (1972) to be 11.7 and $11.9 \mathrm{Ma}(\mathrm{K}-\mathrm{Ar}$ ages). The overlying Ovacık basalt crops out in three different localities around the Urla village as small basaltic lava flows and have been dated by Borsi et al. (1972) to be $11.3 \mathrm{Ma}$ (K-Ar age).

There are some geothermal springs venting on the onshore areas of the Karaburun Peninsula. Çeşme and Gülbahçe are placed in the western part of the İzmir city, where there is a hot water resource of high potential. The waters of this region have surface temperature values ranging between 30 and $60{ }^{\circ} \mathrm{C}$. Çeşme and Gülbahçe water types are of the $\mathrm{Na}-\mathrm{Cl}$ type. The concentration of arsenic $(24 \mu \mathrm{g} / \mathrm{l})$ was measured in the Gülbahçe geothermal fluid in this field.

\section{Discussion}

The Aegean region is well known for its geothermal resources and numerous hot springs. A crustal-scale metamorphic core complex, the Menderes Massif, dissects the approximately east-west grabens to form the most prominent features of the region. In the regional framework, the majority of west Anatolian geothermal waters are of either the $\mathrm{Na}-\mathrm{HCO}_{3}$ or the $\mathrm{Na}-\mathrm{Cl}$ types, although $\mathrm{Na}-\mathrm{SO}_{4}$-type waters are also present. The waters are weakly acidic to alkaline with $\mathrm{pH}$ values ranging from 6.1 to 9.6 , and have total dissolved solids (TDS) contents ranging between 550 and 54,884 ppm (Mutlu and Güleç, 1998). Thermal systems of western Turkey exhibit a wide range of chemical composition that reflects the complex nature and different sources of thermal waters (Table 1). Generally, the four major groups are separately reflecting different origins and mechanisms of waterrock interactions in western Turkey. The first group is an $\mathrm{Na}-\mathrm{Cl}$ type of marine origin found in places such as Cesme, Urla, Seferihisar, and Kestanbol; the second group is an $\mathrm{Na}-\mathrm{HCO}_{3}$ type which is associated with metamorphic rocks of the Menderes Massif. The third group is an $\mathrm{Na}-\mathrm{SO}_{4}$ type that is also associated with metamorphic rocks of the Kazdağı and Menderes Massif and volcanic rocks. The last group is a $\mathrm{Ca}-\mathrm{Mg}-\mathrm{HCO}_{3}-\mathrm{SO}_{4}$ type that results from interactions with carbonate rocks at shallow depths (Vengosh et al., 2002).

Isotopic composition studies have shown that Tuzla, Kavaklıdere, Göbekli, Germencik, and other geothermal fluids in Western Turkey have different origins (Fig. 5). These results also indicate that the hot saline waters (brine) in the Tuzla and Germencik geothermal fields originated from connate water along the faults. Kestanbol, Urla, and Çeşme water samples are easily depicted in Oxygen $\left(\delta^{18} \mathrm{O}\right)$ and Deuterium $\left(\delta^{2} \mathrm{H}\right)$ diagrams to show a mixing type of meteoric or cold water, and sea water. Water from these systems is deep circulated seawater. There is a clear $\left(\delta^{18} \mathrm{O}\right)$ shift from the MMWL. This suggests that the water-rock interaction is an important process for geothermal fluids according to the effect of deep circulation and high temperature of the waters of Tuzla and Germencik. Other geothermal fluids have the same recharge area, shallow circulation, and meteoric in origin as depicted by their $\delta^{18} \mathrm{O}(\%)$ and $\delta^{2} \mathrm{H}(\%)$ data. The age of these all geothermal fluids is more than 50 years as determined by Tritium $\left({ }^{3} \mathrm{H}\right)$ data.

The circulation of the thermal and mineral waters is closely related to major faults and fracture zones. Due to its neotectonic structure and the influence of volcanism, the Anatolian Plate contains various altered rock types that contain elevated levels of arsenic and other trace elements. Most of the thermal waters in Western Turkey are enriched in As with concentrations ranging from 1 to $1419.8 \mu \mathrm{g} / \mathrm{l}$. High arsenic content is attributed to deep water circulation from a deeply seated magma body. The water-rock interaction is the most important source for arsenic. Generally As is attributed to the dissolution of ferromanganese minerals within the Late Miocene sediments in Western Turkey. For example; the high As concentration in the hotsaline waters of the Tuzla Geothermal Field results from the rapid evolution of anoxic conditions in brines (Drever, 1997) and the reduction of the sulfite $\left(\mathrm{SO}_{3}\right)$ formed by reactions with trace elements, followed by release of trace elements during oxidation of the metal sulfides via bacterial processes. Mixing of thermal water and shallow groundwater, (the latter providing the opportunity for oxidation processes to occur), provides an easy pathway for the dissolution of As in an aquatic environment (Baba et al., 2009a, 2009b). The As content may also be elevated, due to the dissolution of As in rhyolite tuff (Stauffer and Thompson, 1984) that is seen in different parts of the study area. Because of factors arising from active tectonics, many ore deposits are exposed along fault systems, where dense alterations can outcrop. Arsenopyrite, orpiment, and realgar can been seen in these zones. For example, Salihli, which is in Gediz Graben, is also known for its mineralized mercury of hydrothermal origin, where small mining operations have been carried out intermittently for several years. The site is currently under reconsideration as a prospect for epithermal mineralized Au-Sb (Larson and Erler, 1993). These ore deposits have arsenopyrite minerals. In the Salihli geothermal field hot springs are concentrated in the Kurşunlu, Kükürtlü, and Sart areas. Therefore high concentrations of As can be seen in the Kurşunlu thermal springs (Yılmazer et al., 2010).

\section{Conclusion}

Most of the geothermal fluids in western Turkey originated from the Menderes Massif Metamorphic, which discharges from the rims 
of the east-west-trending faults that form the Büyük Menderes, Küçük Menderes, Gediz, and Simav grabens. In addition to some thermal springs that originated from Kazdağ 1 Massive in Northwestern Turkey, the circulation of the geothermal fluid is closely related to major faults and fractured zones in these regions. Some of the best known granitoids which are heat sources of the geothermal systems in western Turkey, from north to south, include the following: Karabiga, Kapidag, Armutlu, Kestanbol, Evciler, Eybek, Orhaneli, Topuk, Tepeldag, Kozak, Egrigoz, and Baklan granitoids. Due to its neotectonic structure and the influence of volcanism, the Anatolian Plate contains various altered rock types that contain elevated levels of arsenic. The concentration of arsenic in geothermal waters in many places around western Turkey is found to exceed the maximum allowable limits $(10 \mu \mathrm{g} / \mathrm{l})$ set by national and international standards for drinking water. It is important to monitor arsenic in all geothermal systems and to study in detail the waterrock interactions within each geothermal system in western Turkey.

\section{Acknowledgments}

Dr. Ritchie C. Eanes is acknowledged for his proofreading and suggestions. We also thank Dr. Jochen Bundschuh for the valuable help, and critical review of the manuscript.

\section{References}

Aksoy, N., 2001. Monitoring the Balcova-Narlidere geothermal system using tracers PhD thesis, Dokuz Eylul University Graduate School, Izmir, Turkey, 150 pp.

Aksoy, N., Simşek, C., Gunduz, O., 2009. Groundwater contamination mechanism in geothermal field: a case study of Balcova, Turkey. Journal of Contaminant Hydrology $103,13-28$.

Aldanmaz, E., Pearce, J.A., Thirwall, M.F., Mitchell, J.G., 2000. Petrogenetic evolution of late Cenozoic, post-collision volcanism in western Anatolia, Turkey. Journal of Volcanology and Geothermal Research 102, 67-95.

Altunkaynak, S., Dilek, Y., 2006. Timing and nature of post collisional volcanism in western Anatolia and geodynamic implications. In: Dilek, Y., Pavlides, S. (Eds.) Post-collisional Tectonics and Magmatism in the Mediterranean Region and Asia: Geol. Soc. Am. Special Papers, 409, pp. 321-351.

Arpat, E. Bingol, E., 1969. Ege Bolgesi Graben Sistemi Uzerine Dușunceler. MTA Bulletin 73, 1-9.

Atabey, E., 2009. Arsenic and its effects. Publication of General Directorate of Mineral Research and Exploration (MTA), Ankara. (in Turkish).

Aydogan, M.S., Coban, H., Bozcu, M., Akıncı, O., 2008. Geochemical and mantle-like isotopic (Nd, Sr) composition of the Baklan Granite from the Muratdagi Region (Banaz, Usak), western Turkey: implications for input of juvenile magmas in the source domains of western Anatolia Eocene-Miocene granites. Journal of Asian Earth Sciences 33, 155-176

Baba, A., 2003. Geothermal environmental impact assessment with special reference to the Tuzla, Geothermal Area, Canakkale Turkey (Report 5) Geothermal training in Iceland 2003. UNU-GTP, Iceland, pp. 75-114

Baba, A., 2010. High arsenic levels in water resources resulting from alteration zones: a case study from Biga Peninsula, Turkey. Proceedings of AS2010: The Third International Congress on Arsenic in the Environment, 17-21 May, 2010, Taiwan.

Baba, A., Ármannsson, H., 2006. Environmental impact of the utilization of a geothermal area in Turkey. Energy Sources 1, 267-278.

Baba, A., Deniz, O., 2008. Potential, Determination of Applicability and Evaluation of Environmental Effects of Geothermal Source in the Biga Peninsula, TÜBITAK Project, CAYDAG-104Y082 p. 349 (in Turkish).

Baba, A., Ertekin, C., 2007. Determination of the source and age of the geothermal fluid and its effects on groundwater resources in Kestanbol (Canakkale-Turkey). GO07: Securing Groundwater Quality in Urban and Industrial Environments, 7th International Groundwater Quality Conference,Fremantle, Western Australia, pp. 1-8.

Baba, A. Özcan, H., 2005. Monitoring and evaluation of the geothermal fluid on soil and water in Tuzla geothermal field by GIS. In: Erasmi, S., Cyffka, B., Kappas, M. (Eds.) Remote sensing and GIS for environmental studies: Göttinger Geographische Abhandlungen, vol. 113, pp. 138-143 (Göttingen).

Baba, A., Özcan, H., Deniz, O., 2005. Environmental impact by spill of geothermal fluids at the geothermal field of Tuzla, Canakkale-Turkey. Proceedings of the World Geothermal Congress 2005 Antalya, Turkey (8 pp.)

Baba, A., Yuce, G., Denız, O., Ugurluoglu, Y.D., 2009a. Hydrochemical and isotopic composition of Tuzla geothermal (Canakkale-Turkey) field and its environmental impacts. Journal of Environmental Fresenius 10, 144-161.

Baba, A., Deniz, O., Ozcan, H., Erees, S.F., Cetiner, S.Z., 2009b. Geochemical and radionuclide profile of Tuzla geothermal field, Turkey. Environmental Monitoring and Assessment 145, 361-374

Baba, A., Save, D., Gündüz, O., Gürdal, G., Bozcu, M., Sülün, S., Özcan, H., Hayran, O. Íkiısık, H., Bakırcı, L., 2009c. The Assessment of Mining Activities in Can Coal District from Medical Geology Perspective. CAYDAG-106Y041 p. 479 (in Turkish).
Ball, J.W., Nordstrom, D.K., Jenne, E.A., Vivit, D.V., 1998. Chemical analysis of hot springs, pools, geysers, and surface waters from Yellowstone National Park, Wyoming and its vicinity. USGS Open-File Report, 98, p. 182.

Borsi, J., Ferrara, G., Innocenti, F., Mazzuoli, R., 1972. Geochronology and petrology of recent volcanics in the easthern Aegean Sea (West Anatolia and Lesbos Island). Bulletin of Volcanology 36, 473-496.

Bozkurt, E., 2000. Timing of extension on the Büyük Menderes Graben, western Turkey, and its tectonic implications. In: Bozkurt, E., Winchester, J.A., Piper, J.D.A. (Eds.), Tectonics and Magmatism in Turkey and The Surrounding Area: Geological Society, London, Special Publications, 173, pp. 385-403.

Bozkurt, E., 2001a. Neotectonics of Turkey-a synthesis. Geodinamica Acta 14, 3-30.

Bozkurt, E., 2001b. Late Alpine evolution of the central Menderes Massif, western Turkey. International Journal of Earth Sciences, http://dx.doi.org/10.1007/s005310000141.

Bozkurt, E., 2003. Origin of NE-trending basins in western Turkey. Geodinamica Acta $16,61-81$.

Bozkurt, E., Oberhänslı, R., 2001. Menderes Massif (Western Turkey): structural, metamorphic and magmatic evolution: a synthesis. International Journal of Earth Sciences 89, 679-708.

Bozkurt, E., Park, R.G., 1994. Southern Menderes Massif-an incipient metamorphic core complex in Western Anatolia, Turkey. Journal of the Geological Society of London $151,213-216$.

Bulbul, A., 2009. Hydrogeological and hydrogeochemical assessment of cold and hot water systems of Alasehir (Manisa). PhD Thesis, Dokuz Eylul University Graduate School of Natural and Applied Sciences, Izmir, Turkey. (in Turkish).

Bulut, M., Filiz, S., 2005. Hydrogeology, hydrochemistry and isotopic properties of Bayındır Geothermal Field (Izmir, Western Anatolia, Turkey). MTA Journal 131, 63-78 (in Turkish)

Bundschuh, J., Bhattacharya, P., Hoinkis, J., Kabay, N., Jean, J.S., Litter, M.I., 2010. Groundwater arsenic: from genesis to sustainable remediation. Water Research $44,5511$.

Çakmakoğlu, A., Bilgin, Z.R., 2006. Pre-Neogene stratigraphy of the Karaburun Peninsula ( $W$ of İzmir Turkey). Bulletin of the Mineral Research and Exploration Institute of Turkey 132, 33-61.

Cohen, H.A., Dart, C.J., Akyüz, H.S., Barka, A., 1995. Syn-Rift sedimentation and structural development of the Gediz and Büyük-Menderes Graben, Western Turkey. Journal of the Geological Society of London 152, 629-638.

Colak, M., Gemici, U., Tarcan, G., 2003. The effects of colemanite deposits on the arsenic concentrations of soil and groundwater in Igdeköy-Emet, Kütahya, Turkey. Water, Air, and Soil Pollution 149, 127-143.

Conrad, M.A., Hipfel, B., Satir, M., 1995. Chemical and stable isotopic characteristics of thermal waters from the Cesme-Seferihisar area, Izmir (W. Turkey). IESCA, 2, pp. 669-679.

Deniz, O., Baba, A., Tarcan, G., 2010. Hydrogeochemical and hydrological investigation of the Can Geothermal Field. Geological Bulletin of Turkey 53 (2-3), 159-184.

Dilek, Y., Altunkaynak, S., 2007. Cenozoic crustal evolution and mantle dynamics of post-collisional magmatism in western Anatolia. International Geology Review $49,431-453$.

Dilsiz, C., 2006. Conceptual hydrodynamic model of the Pamukkale hydrothermal field, southwestern Turkey, based on hydrochemical and isotopic data. Hydrogeology Journal 14 (4), 562

Dogan, M., Dogan, A.U., 2007. Arsenic mineralization, source, distribution, and abundance in the Kutahya region of the western Anatolia, Turkey. Environmental Geochemistry and Health 29, 119-129.

Drever, I., 1997. The Geochemistry of Natural Waters, Surface and Groundwater Environments, 3rd ed. Prentice Hall, Upper Saddle River, NJ, 07458, pp. 164-178.

Ellis, A.J., Mahon, W.A.J., 1967. Natural hydrothermal systems and experimental hot water/rock interactions. Geochimica et Cosmochimica Acta 31, 519-538.

Emre, T., 1996. Gediz grabeninin jeolojisi ve tektoniği (Geology and tectonics of the Gediz graben). Turkish Journal of Earth Sciences 5, 171-185.

Emre, T., Sözbilir, H., 1997. Field evidence for metamorphic core complex, detachment faulting and accommodation faults in the Gediz and Büyük Menderes grabens (Western Turkey). International Earth Science Colloquium on the Aegean Region (IESCA-95), 1, pp. 73-94.

Emre, T., Sözbilir, H., 2007. Tectonic Evolution of the Kiraz Basin, Küçük Menderes Graben: evidence for compression/uplift-related basin formation overprinted by extensional tectonics in West Anatolia. Turkish Journal of Earth Sciences 16, 441-470.

Erdoğan, B., 1990. Stratigraphic features and tectonic evolution of the İzmir-Ankara Zone located between İzmir and Seferihisar. Turkish Association of Petroleum Geologists (TPJD) Bulletin 2, 1-20.

Erisen, B., Can, A.R., Yıldırım, N., 1989. Simav Eynal (Kutahya) Geothermal Site and Evaluation of Wells (EJ1 and EJ2) Report, MTA, No:8916, Ankara (in Turkish).

Erkül, F., Helvacı, C., Sözbilir, H., 2005. Evidence for two episodes of volcanism in the Bigadic borate basin and tectonic implications for western Turkey. Geological Journal 40, 1-16.

Ersoy, E.Y., Helvacı, C., Sözbilir, H., Erkül, F., Bozkurt, E., 2008. A geochemical approach to Neogene-Quaternary volcanic activity of Western Anatolia: an example of episodic bimodal volcanism within the Selendi Basin, Turkey. Chemical Geology 255, 265-282.

Filiz, S., 1982. Investigation of Aegean Region Geothermal System with ${ }^{18} \mathrm{O},{ }^{2} \mathrm{H},{ }^{3} \mathrm{H},{ }^{13} \mathrm{C}$ with isotopes. Ege University, Assoc. Prof. Thesis, p. 95. Izmir (in Turkish)

Fytikas, M., Innocenti, F., Manetti, P., Mazzuoli, R., Peccerillo, A., Villari, L., 1984. Tertiary to Quaternary evolution of volcanism in the Aegean region. In: Dixon, JE. Robertson, A.H.F. (Eds.), The Geological Evolution of the Eastern Mediterranean: Geol. Soc. Spec. Publ., 17, pp. 687-699.

García-Veigas, J., Rosell, L., Federico, Ortí, Gündoğan, İ., Helvacı, C., 2011. Mineralogy, diagenesis and hydrochemical evolution in a probertite-glauberite-halite saline lake (Miocene, Emet Basin, Turkey). Chemical Geology 280 (3-4), 352-364. 
Gemici, U., 2008. Evaluation of the water quality related to the acid mine drainage of an abandoned mercury mine (Alaşehir, Turkey). Environmental Monitoring and Assessment 147, 93-106.

Gemici, U., Filiz, S., 2001. Hydrochemistry of the Cesme geothermal area, Turkey. Journal of Volcanology and Geothermal Research 110,171-188.

Gemici, U., Tarcan, G., 2001. Hydrogeochemistry of the Saraycik geothermal area (Manisa) Western Turkey. Water-Rock Interaction 2001, Villasimus, Italy, 10-15 June 2001, Proceedings, vol. 2, pp. 831-834.

Genç, S.C., Altunkaynak, S.., Karacık, Z., Yılmaz, Y., 2001. The Çubukludağ Graben, Karaburun peninsula: it's tectonic significance in the Neogene geological evolution of the western Anatolia. Geodinamica Acta 14, 45-55.

Gessner, K., Rıng, U., Chrıstopher, J., Hetzel, R., Passchır, C.W., Güngör, T., 2001. An active bivergent rolling-hinge detachment system: central Menderes metamorphic core complex in western Turkey. Geology 29, 611-614.

Gokgoz, A., Yilmazli, I.E., Gungor, I., Yavuzer, I., 2010. Hydrogeology and environmental study at the Karahayit geothermal field (Western Turkey). Proceedings World Geothermal Congress 2010, Bali, Indonesia, 25-29 April 2010.

Gonzalez-Partida, E., Hinojosa, T.E., Verma, M.P., 2001. Interraccion agua geothermicamanantiales en el campo geotermico de Los Humeros, Mexico. Ingenieria Hidraulica en Mexico, XVI, pp. 185-194.

Gulec, N., 1988. Helium-3 distribution in Western Turkey. MTA Bulletin 108, 35-42.

Gunduz, O., Baba, A., Elpit, H., 2010a. Arsenic in groundwater in Western Anatolia, Turkey: a review. XXVIII IAH Congress, Groundwater Quality Sustainability,12-17 September 2010, Krakow, Poland, pp. 183-191.

Gunduz, O., Simsek, C., Hasozbek, A., 2010b. Arsenic pollution in the groundwater of Simav Plain, Turkey: its impact on water quality and human health. Water, Air and Soil Pollution 205, 43-62.

Güneş, C., 2006. Evaluation of hyrogeological and hydrogeochemical study of the Gediz Spas, Kütahya, Turkey, Ms. Thesis, DEU, Izmir (in Turkish).

Güngör, T., Erdoğan, B., 2002. Tectonic significance of mafic volcanic rocks in a Mesozoic sequence of the Menderes Massif, west Turkey. International Journal of Earth Sciences 91, 386-397.

Helvacı, C., 1986. Startigraphic and structural evolution of the Emet borate deposits, Western Anatolia. DEU Research Paper No: MM /jeo-86 ar 008. (28 pp. (in Turkish)).

Helvacı, C., Alonso, R.N., 2000. Borate deposits of Turkey and Argentina; a summary and geological comparison. Turkish Journal of Earth Sciences 24, 1-27.

Helvacı, C., Orti, F., 1998. Sedimantology and diagenesis of Miocene colemantite-ulexite deposits (Western Anatolia, Turkey). Journal of Sedimentary Research 68 (5), 1021-1033.

Hetzel, R., Ring, U., Akal, C., Troesch, M., 1995. Miocene NNE-directed extensional unroofing in the Menderes Massif, southwestern Turkey. Journal of the Geological Society of London 152, 639-654.

Hinsbergen, D.J.J., Kaymakci, N., Spakman, W., Torsvik, T.H., 2010. Reconciling the geological history of western Turkey with plate circuits and mantle tomography. Earth and Planetary Science Letters 297, 674-686.

Ilkisik, O.M., 1995. Regional heat flow in western Anatolia using silica temperature estimates from thermal springs. Tectonophysics 244, 175-184.

Işı, V., Tekeli, O., Seyitoğlu, G., 2004. The Ar-40/Ar-39 age of extensional ductile deformation and granitoid intrusion in the northern Menderes core complex: implications for the initiation of extensional tectonics in Western Turkey. Journal of Asian Earth Sciences 23, 555-566.

Karacık, Z., Yılmaz, Y., 2000. Dikili Çandarlı bölgesinin jeolojik ve genç tektonik özellikleri. BADSEM Sempozyumu, Izmir, pp. 483-486.

Karacık, Z., Yılmaz, Y., Pearce, J.A., 2007. The Dikili-Çandarlı volcanics, Western Turkey: magmatic interactions as recorded by petrographic and geochemical features. Turkish Journal of Earth Sciences 16, 493-522.

Karamanderesi, I.H., 1997. Geology and hydrothermal alteration processes of the Salavatll-Aydın geothermal field. PhD Thesis, Dokuz Eylul University, Izmir, Turkey.

Kasap, I., 1984. Geothermal resource evaluation of Germencik-Omerbeyli (Western Anatolia) geothermal field of Turkey. Seminar on Utilization of Geothermal Energy for Electric Power Production and Space Heating, 14-17 May, Florence.

Kaya, O., 1981. Miocene reference section for the coastal parts of West Anatolia. Newsletters on Stratigraphy 10, 164-191.

Kaya, O., Ünay, E., Saraç, G., Eıchhorn, S., Hassenrück, S., Knappe, A., Pekdeğer, A., Mayda, S., 2004. Halitpaşa transpressive zone: implications for an Early Pliocene compressional phase in central western Anatolia, Turkey. Turkish Journal of Earth Sciences 13,1-13.

Kaya, O., Ünay, E., Göktaş, F., Saraç, G., 2007. Early Miocene stratigraphy of Central West Anatolia, Turkey: implications for the tectonic evolution of the Eastern Aegean area. Geological Journal 42, 85-109.

Koçyiğit, A., Yusufoğlu, H., Bozkurt, E., 1999. Evidence from the Gediz graben for episodic two-stage extension in Western Turkey. Journal of the Geological Society of London 156, 605-616.

Koralay, O.E., Satir, M., Dora, O.O., 2001. Geochemical and geochronological evidence for Early Triassic calk-alkaline magmatism in the Menderes Massif, western Turkey. International Journal of Earth Sciences 89 (4), 822-835.

Larson, L.T., Erler, A., 1993. The epithermal lithogeochemical signature-a persistent characterization of precious metal mineralization at Kursunlu and Örencik, two prospects of very different geology in western Turkey. Journal of Geochemical Exploration 47, 321-331

Lips, A.L.W., Cassard, D., Sözbilir, H., Yılmaz, H., Wijbrans, J., 2001. Multistage exhumation of the Menderes Massif, western Anatolia (Turkey). International Journal of Earth Sciences 89, 781-792.

Magri, F., Akar, T., Gemici, U., Pekdeger, A., 2010. Deep geothermal groundwater flow in the Seferihisar-Balcova area, Turkey: results from transient numerical simulations of coupled fluid flow and heat transport processes. Geofluids 10, 3,388-3,405.
Motyka, R.J., Poreda, R.J., Jeffrey, A.W.A., 1989. Geochemistry, isotopic composition, and origin of fluids emanating from mud volcanoes in the Copper River basin, Alaska. Geochimica et Cosmochimica Acta 53, 3302-3309.

MTA, 1980. Hot and mineral water inventory. General Directorate of Mineral Research and Exploration (MTA). (MTA Rap., Ankara).

MTA, 2002. Geological Map of Turkey (1:500000). Publication of Mineral Research and Exploration Institute of Turkey.

MTA-JICA, 1987. Pre-feasibility study on the Dikili Bergama geothermal development project in the Republic of Turkey. Progress Report II, Final Report. M.T.A., Ankara.

Mutlu, H., 1998. Chemical geothermometry and fluid-mineral equilibria for the OmerGecek thermal waters, Afyon area, Turkey. Journal of Volcanology and Geothermal Research 80 (3-4), 303-321.

Mutlu, H., 2007. Constraints on the origin of the BalÝkesir thermal waters (Turkey) from stable isotope $\left(\mathrm{d}^{18} \mathrm{O}, \mathrm{dD}, \mathrm{d}^{13} \mathrm{C}, \mathrm{d}^{34} \mathrm{~S}\right)$ and major-trace element compositions Turkish Journal of Earth Sciences 16, 13-32.

Mutlu, H., Güleç, N., 1998. Geochemical characteristics of thermal waters from Anatolia (Turkey). Journal of Volcanology and Geothermal Research 85, 495-515.

Mützenberg, S., 1997. Nature and origin of the thermal springs in the Tuzla area, Western Anatolia, Turkey. In: Schindler, C., Pfister, M. (Eds.), The Marmara Poly-Project. Vdf Hochschulverlag AG an der ETH, Zurich, pp. 301-317.

Nordstrom, D.K., 2010. Geothermal Arsenic, Arsenic in Geosphere and Human Diseases. In: Jean, Bunddschuh, Bhattacharya (Eds.), Taylor \& Francis Group, London, pp. 41-43.

Oberhänsli, R., Partzsch, J., Candan, O., Çetinkaplan, M., 2001. First occurrence of Fe$\mathrm{Mg}$-carpholite documenting a high-pressure metamorphism in metasediments of the Lycian Nappes, SW Turkey. International Journal of Earth Sciences 89 (4), 867-873.

Ocakoğlu, N., Demirbağ, E., Kuşcu, İ., 2004. Neotectonic structures in the area offshore of Alaçatı, Doğanbey and Kuşadası (western Turkey): evidence of strike-slip faulting in the Aegean extensional province. Tectonophysics 391, 67-83.

Okay, A., 2001. Stratigraphic and metamorphic inversions in the central Menderes Massif: a new structural model. International Journal of Earth Sciences 89, 709-727.

Okay, A.İ., Altıner, D., 2004. A condensed Mesozoic succession North of İzmir: a fragment of the Anatolide-Tauride Platform in the Bornova Flysch Zone. Turkish Journal of Earth Sciences 16, 257-279.

Okay, A.I., Altıner, D., 2007. A condensed Mesozoic section in the Bornova Flysch Zone: a fragment of the Anatolide-Tauride carbonate platform. Turkish Journal of Earth Sciences 16, 257-279.

Okay, A.İ., Satır, M., 2000. Coeval plutonism and metamorphism in a latest Oligocene metamorphic core complex in northwest Turkey. Geological Magazine 5, 495-516.

Okay, A.I.., Siyako, M., 1991. The new position of the İzmir-Ankara Neo-Tethyan suture between İzmir and Balıkesir. Proceedings of the Ozan Sungurlu Symposium, pp. $333-355$.

Okay, A.İ., Tüysüz, O., 1999. Tethyan sutures of northern Turkey. In: Durand, B., Jolivet, L., Hovarth, F., Séranne, M. (Eds.), The Mediterranean basins: Tertiary extension within the Alpine orogenJournal of the Geological Society of London 156, 475-515

Okay, A.I., Satır, M., Maluski, H., Sıyako, M., Monıe, P., Metzger, R., Akyüz, S., 1996 Paleo- and Neo-Tethyan events in northwest Turkey: geological and geochronological constraints. In: Yın, A., Harrison, M. (Eds.), Tectonics of Asia. Cambridge University Press, Cambridge, pp. 420-441.

Okay, A.I.., Tansel, İ., Tüysüz, O., 2001. Obduction, subduction and collision as reflected in the Upper Cretaceous-Lower Eocene sedimentary record of Western Turkey Geological Magazine 138, 117-142.

Ozen, T., Bulbul, A., Tarcan, G., 2010. Reservoir and hydrogeochemical characterizations of the Salihli geothermal fields in Turkey. Proceedings World Geothermal Congress 2010, Bali, Indonesia, 25-29 April 2010.

Ozer, S. Sözbilir, H, 2003. Presence and tectonic significance of Cretaceous rudist species in the so-called Permo-Carboniferous Göktepe Formation, central Menderes metamorphic masif, western Turkey. International Journal of Earth Sciences 92, 97-404.

Ozgenç, I., Ilbeyli, N., 2008. Petrogenesis of the Late Cenozoic Eğrigöz Pluton in Western Anatolia, Turkey: implications for magma genesis and crustal processes. International Geology Review 50, 375-391.

Ozgur, N., 2002. Geochemical signature of Kızıldere geothermal field, Western Anatolia, Turkey. International Geology Review 44 (2), 153-163.

Ozgur, N., Halbach, P., Pekdeger, A., Sonmez, N., Dora, O.Ö., Ma, D.-S., Wolf, M., Stichler W., 1997. Epithermal antimony, mercury, and gold deposits in the rift zone of the Kücük Menderes, Western Anatolia, Turkey: preliminary studies. In: Papunen, $\mathrm{H}$ (Ed.), Mineral Deposits: Research and Exploration, Where do They Meet? : 4th Biennnial SGA Meeting, Turku, Finland, pp. 269-272.

Özkaymak, C., Sözbilir, H., 2008. Stratigraphic and structural evidence for fault reactivation: the active Manisa fault zone, western Anatolia. Turkish Journal of Earth Sciences 17 (3), 615-635.

Ozturk, Y.Y., Helvaci, C., 2008. Skarn Alteration and Au-Cu Mineralization Associated with Tertiary Granitoids in Northwestern Turkey: evidence from the Evciler Deposit, Kazdag Massif, Turkey. Economic Geology 103, 1665-1682.

Palabiyik, Y., 2006. Kutahya-Simav Jeotermal Sahası'nın jeokimyasal değerlendirilmesi, ITU, Ms. Thesis (in Turkish).

Palabiyik, Y., Serpen, U., 2008. Geochemical assessment of Simav geothermal field, Turkey. Revista Mexicana de Ciencias Geológicas 25 (3), 408-425.

Palmer, M.P., Helvacı, C., Fallick, A.E., 2009. Sulphur, sulphate oxygen and strontium isotope composition of Cenozoic Turkish evaporites. Chemical Geology 209, 341-356.

Pe-Piper, G., Piper, D.J.W., 2001. Late Cenozoic, post-collisional Aegean igneous rocks: $\mathrm{Nd}, \mathrm{Pb}$ and $\mathrm{Sr}$ isotopic constraints on petrogenetic and tectonic models. Geologica Magazine 138, 653-668. 
Pe-Piper, G., Piper, D.J.W., Kotopouli, C.N., Panagos, A.G., 1995. Neogene volcanoes of Chios, Greece: the relative importance of subduction and back-arc extension. Geological Society London Special Publication 81, 213-232.

Purvis, M., Robertson, A., 2004. A pulsed extension model for the Neogene-Recent EW-trending Alasehir Graben and the NE-SW-trending Selendi and Gördes Basins, Western Turkey. Tectonophysics 391, 171-201.

Ring, U., Susanne, L., Matthias, B., 1999. Structural analysis of a complex nappe sequence and late orogenic basins from the Agean Island of Samos, Greece. Journa of Structural Geology 21, 1575-1601.

Robertson, A.H.F., Pickett, E.A., 2000. Paleozoic-Early Tertiary Tethyan evolution of mélanges, rift and passive margin units in the Karaburun Peninsula (western Turkey) and Chios Island (Greece). In: Bozkurt, E., Winchester, J.A., Piper, J.D.A. (Eds.), Tectonics and Magmatism in Turkey and Surrounding Area: Geol. Soc. London, Spec. Publ., 173, pp. 43-82.

Romero, L, Alonso, H., Campano, P., Fanfani, L Cidu, R., Dadea, C. Keegan, T. Thornton, I. Farago, M., 2003. Arsenic enrichment in waters and sediments of the Rio Loa (second region, Chile). Applied Geochemistry 18 (9), 1399-1416.

Samilgil, E., 1966. Hydrogeological Report of Geothermal Energy Possibility Survey of Hot Springs of Kestanbol and Tuzla village of Çanakkale. MTA, Ankara. (report 4274).

Samilgil, E., 1985. Jeotermal uygulamalarda cevre sorunları. Üniversitesi Mühendislik Fakültesi Yerbilimleri Dergisi 5 (1-2), 1-106 (in Turkish).

Sanlıyüksel, D., Baba, A., 2007. Hydrogeochemical and isotopic study of Kırkgeçit (BigaÇanakkale) geothermal area. Proceedings of the 60th Geological Congress, Ankara, pp. 89-92.

Sari, C., Salk, M., 2003. Heat flow investigations in Western Anatolia. Geophysical Research Abstracts, 5, p. 08509 (EGS-AGU-EUG Joint Assembly, Nice, France)

Schuiling, R.D., 1962. On petrology, age and evolution of the Menderes Massif, W Turkey: a rubidium/strontium and oxygen isotope study. Bulletin of the Minera Research and Exploration Institute of Turkey 58, 703-714.

Şengör, A.M.C., Yılmaz, Y., 1981. Tethyan evolution of Turkey: a plate tectonic approach. Tectonophysics 75, 181-241.

Sengör, A.M.C., Satır, M., Akkök, R., 1984. Timing of tectonic events in the Menderes Massif, Western Turkey: implications for tectonic evolution and evidence for Pan-African basement in Turkey. Tectonics 3, 693-707.

Sengör, A.M.C., Görür, N., Saroğlu, F., 1985. Strike-slip faulting and related basin formation in zones of tectonic escape: Turkey as a case study. In: Biddle, K., Chrıstıe-Blıck, N. (Eds.), Strike-Slip Deformation, Basin Formation and Sedimentation: Society of Economic Paleontologists and Mineralogists, Special Publications, 37, pp. 227-264.

Seyitoğlu, G., 1997. Late Cenozoic tectono-sedimentary development of Selendi and Uşak-Güre basins: a contribution to the discussion on the development of east-west and north-trending basins in western Turkey. Geological Magazine 134, 163-175.

Seyitoğlu, G., Scott, B.C., 1992. Late Cenozoic volcanic evolution of the northeastern Aegean region. Journal of Volcanology and Geothermal Research 54, 157-176.

Seyitoğlu, G., Scott, B., 1996. The age of the Alașehir graben (west Turkey) and its tectonic implications. Geological Journal 31, 1-11.

Seyitoglu, G., Anderson, D., Nowell, G., Scott, B., 1997. The evolution from Miocene potassic to Quaternary sodic magmatism in western Turkey: implications for enrichment processes in the lithospheric mantle. Journal of Volcanology and Geothermal Research 96, 127-147.

Seyitoğlu, G., Tekeli, O., Cemen, I., Sen, S., Işık, V., 2002. The role of the flexural rotation/ rolling hinge model in the tectonic evolution of the Alaşehir Graben, western Turkey. Geological Magazine 139, 15-26.

Simsek, S., 1984. Aydin-Germencik-Omerbeyli geothermal field of Turkey. Seminar on Utilisation of Geothermal Energy for Electric Power Production and Space Heating, 14-17 May 1984, Florence. (Sem. ref. no EP/SEM.9/R.37).

Simsek, S., 2003. Hydrogeological and isotopic survey of geothermal fields in the Buyuk Menderes Graben, Turkey. Geothermics 32, 669-678.

Simsek, C., 2010a. Boron and arsenic contamination in Balçova geothermal field TESCO2010. Geothermal Energy Seminer, pp. 361-368 (in Turkish).

Simsek, S., 2010b. Exploration experiences on geothermal energy in Turkey some case studies. Geothermal Congress, Der Geothermiekongress 2010, 17-19 November, 2010 Karlsruhe-Germany, pp. 1-9.

Simsek, S. Yildirim, N., Simsek, Z.N., Karakus, H., 2002. Changes in geotherma resources at earthquake regions and their importance. Proceedings of Middle Anatolian Geothermal Energy and Environmental Symposium, pp. 1-13.

Siyako, M., Burkan, K.A., Okay, A.l., 1989. Biga ve Gelibolu yarımadalarının Tersiyer jeolojisi ve hidrokarbon olanakları. Türkiye Petrol Jeologlari Dernegi Bülteni 1 183-199.

Sözbilir, H., 2001. Extensional tectonics and the geometry of related macroscopic structures: field evidence from the Gediz detachment, western Turkey. Turkish Journal of Earth Sciences 10, 51-67.

Sözbilir, H., 2002. Geometry and origin of folding in the Neogene sediments of the Gediz Graben, western Anatolia, Turkey. Geodinamica Acta 15, 277-288.

Sözbilir, H., Emre, T., 1990. Neogene stratigraphy and structure of the northern rim of the Büyük Menderes graben. Proceedings of International Earth Science Colloquium on the Aegean Region, 2, pp. 314-322.

Sözbilir, H., Erkül, F. Sümer, Ö., 2003. Field evidence for post-Miocene NE-trending accomodation zone lying between Gümüldür (İzmir) and Bigadiç (Balıkesir), west Anatolia. Geological Congress of Turkey, Ankara, Abstracts, pp. 85-86.
Sözbilir, H., Uzel, B., Sümer, Ö., İnci, U., Ersoy, Y., Kocer, T., Demirtas, R., Özkaymak, C., 2008. Evidence for a kinematically linked E-W trending İzmir Fault and NEtrending Seferihisar Fault: kinematic and paleoseismogical studies carried out on active faults forming the İzmir Bay, Western Anatolia. Türkiye Jeoloji Bülteni 51 (2), 91-114.

Sözbilir, H., Sarı, B., Uzel, B., Sümer, Ö., Akkiraz, S., 2011. Tectonic implications of transtensional supradetachment basin development in an extension-parallel transfer zone: the Kocaçay Basin, western Anatolia, Turkey. Basin Research 23, 423-448.

Stauffer, R.E., Thompson, J.M., 1984. Arsenic and antimony in geothermal waters of Yellowstone National Park, Wyoming, USA. Geochimica et Cosmochimica Acta $48,2547-2561$

Tarcan, G., 1995. Hydrogeological study of the Turgutlu Hot Springs. PhD Thesis, Dokuz Eylul University, Graduate School of Natural and Applied Sciences, Izmir

Tarcan, G., 2000. Hydrogeology and Hydrogeochemistry of the Gülbahçe Bay Hydrothermal Karst System, Izmir, Turkey. International Symposium and Field Seminar on, Present State and Future Trends of Karst Studies, Sept. 17-26 Marmaris-Turkey.

Tarcan, G., Filiz, S., 1997. Hydrogeology of the Turgutlu geothermal field. Turk Earth Sci $6-2,43-64$

Tarcan, G., Gemici, U., 2001. Hydrogeochemistry of the Gumuskoy and Sazlikoy geothermal fields, Aydin, Turkey. Water-Rock interaction 2001, Villasimus, vol. 2, Italy, 10-15 June 2001, Proceedings, pp. 931-934.

Tarcan, G., Gemici, Ü., 2003. Water chemistry of the Seferihisar geothermal area, Izmir, Turkey. Journal of Volcanology and Geothermal Research 126, 225-242.

Tarcan, G., Gemici, Ü., 2005. Effects of the contaminants from Turgutlu-Urganlı thermomineral waters on the cold ground and surface waters. Bulletin of Environmental Contamination and Toxicology 74/3, 485-492.

Tarcan, G., Gemici, Ü., 2010. Hydrogeochemical Assessment of the Bergama Thermal Waters, İzmir, Turkey. Proceedings World Geothermal Congress 2010, Bali, Indonesia, 25-29 April 2010, pp. 1-8.

Tarcan, G., Filiz, S., Gemici, U., 2000. Geology and geochemistry of the Salihli geothermal fields, Turkey. Proceedings of WGC-2000 World Geothermal Congress 28 May-10 June, 2000 Congress Kyushu-Tohoku, Japan, pp. 1829-1834.

Tarcan, G., Gemici, U., Aksoy, N., 2005. Hydrogeological and geochemical assessments of the Gediz Graben geothermal areas, Western Anatolia, Turkey. Environmental Geology 47 (4), 523-534.

Tatar-Erkül, S., Sözbilir, H., Erkül, F., Helvacı, C., Ersoy, E.Y., Sümer, Ö., 2008. Geochemistry of I-type granitoids in the Karaburun Peninsula, West Turkey: evidence for Triassic continental arc magmatism following closure of the Palaeotethys. Island Arc 17, 394-418.

Tokçaer, M., 2007. Batı anadolu jeotermal akıșkanlarında borun jeokimyasal döngüsü ve izotop fraksiyonlaşması, Dokuz Eylül Üniversitesi Fen Bilimleri Enstitüsü Doktora Tezi Jeoloji Mühendisliği Bölümü, Ekonomik Jeoloji Anabilim Dalı, pp. 183.

Uzel, B., Sözbilir. H., 2006. An example of basins which developed under the control of normal faults and strike-slip faults: Cumaovası pull-apart basin, İzmir. 10th Meeting of Active Tectonics Research Group, Abstracts, p. 93.

Uzel, B., Sözbilir, H., 2008. A First record of strike-slip basin in western Anatolia and its tectonic implication: the Cumaovası basin as an example. Turkish Journal of Earth Sciences 17, 559-591.

Van Halem, D., Bakker, S.A., Amy, G.L., van Dijk, J.C., 2009. Arsenic in drinking water: a worldwide water quality concern for water supply companies. Drinking Water Engineering and Science 2, 29-34.

Vengosh, A., Helvacı, C., Karamanderesi, I.H., 2002. Geochemical constrains for the origin of thermal waters from western Turkey. Applied Geochemistry 17 (3), $163-183$.

Yalcın, T., 2007. Geochemical Characterization of the Biga Peninsula Thermal Waters (NW Turkey). Aquatic Geochemistry 75-93.

Yigitbas, E., Elmas, A., Sefunc, A., Ozer, N., 2004. Major neotectonic features of eastern Marmara region, Turkey: development of the Adapazari-Karasu corridor and its tectonic significance. Geological Journal 39 (2), 179-198.

Yildirim, N., Önce, S., Akman, U.A., 2010a. High Enthalpy Geothemal Potential Possibility in SW of Büyük Menderes Graben, Turkey. Proceedings World Geothermal Congress 2010 Bali, Indonesia, 25-29 April 2010.

Yildirim, N., Önc, S., Unal, A., 2010b. Geochemical Investigation in Areas Without Geothermal Surface Geothermal Manifestations Kucuk Menderes Graben Western Turkey. Proceedings World Geothermal Congress 2010 Bali, Indonesia, 25-29 April 2010.

Yılmaz, Y., Genç, S.C., Gürer, F., Bozcu, M., Yılmaz, K., Karacık, Z., Altunkaynak, S., Elmas, A. 2000. When did the Western Anatolian grabens begin to develop? In: Bozkurt E., Winchester, J.A., Piper, J.A.D. (Eds.), Tectonics and Magmatism in Turkey and the Surrounding Area: Journal of Geological Society of London, 173, pp. 131-162.

Yllmazer, S., 1989. Geochemical features of Balçova hot springs and geothermal energy possibilities for the area. $\mathrm{PhD}$ thesis, Akdeniz University, Graduate School, Isparta, Turkey.

Yllmazer, S., Pasvanoğlu, S., Vural, S., 2010. The relation of geothermal resources with young tectonics in the Gediz Graben (West Anatolia, Turkey) and their hydrogeochemical analyses. Proceedings World Geothermal Congress 2010 Bali, Indonesia, 25-29 April 2010. 